\title{
Local Instability Theory in Convective Cloud
}

\author{
by \\ Yoshimasa Takaya* \\ Meteorological Research Institute, Tsukuba, Ibaraki, 305 Japan
}

(Received February 18, 1989; Revised December 1, 1989)

\begin{abstract}
A linear instability theory in clouds is developed to explain both the formation mechanism of the uneven structure on the surface of the convective cloud and the mixing or entrainment process.

This instability is a generalization of the conditional instability which is usually discussed in a stratification which is static and horizontally uniform.

In clouds, on the other hand, the stratification of pressure, temperature, water-vapor mixing ratio and cloud water mixing ratio are tilted against the horizon and the wind blows.

At the same time, a simple model of the parameterization of eddy effect inside a cloud is proposed by using this instability theory.
\end{abstract}

\section{Introduction}

Recently the entrainment or mixing process in clouds are extensively studied by observation (Paluch and Baumgardner, 1989) and many models for the process have been exposed to severe criticism.

The mechanism of the entrainment or mixing in convective clouds is much different from that of thermals or plumes in laboratory experiments. This comes from the essential difference in the nature of the boundary. The boundary for thermals or plumes separates fluids of different densities as well as different momentum. Accordingly the shear is usually very large at the boundary (Turner, 1986).

In the case of the convective cloud in the atmosphere, however, the boundary is recognized by the difference in the phase of water. Therefore the usual picture of the engulfment

*) Present affiliation: Office of Archives and Library, Japan Meteorological Agency, 1-3-4, Ohte-machi, Chiyoda-ku, Tokyo, 100 Japan. of the surrounding matter being achieved through highly nonlinear billows may not be appropriate as a model of the entrainment or mixing in the convective cloud. In fact, the recent observation in clouds by Paluch and Baumgardner (1989) denied the existence of such billows.

In this paper, we show that there exists a certain unstable wave inside of a cloud which is a generalized conditional instability.

In this case, the stratification of pressure, temperature, water-vapor mixing ratio and cloud-water mixing ratio are tilted against the horizon and the wind blows. Therefore our study is about the stability of the moving system and not a statically balanced system.

The mechanism of this instability is shown to have three constituents. The first is the effect of the buoyancy whose direction is vertical. The second is the effect of the tilted stratification of the potential temperature, the water-vapour and the cluod-water in cloud, by which discrepancy arises between the direction of the buoyancy (vertical) and the direction in 
which the saturated wet air parcel gets the maximum increase of buoyancy. The third comes into eigenvalue equation in the form of divergence of the mean velocity, but its effect is negligibly small.

This instability produces heterogeneous distribution of the cloud-water. Besides, the more cloudy region moves outward and the less cloudy one inward. This motion forms the uneven structure of the surface of the cloud, which should be compared with the usual billow-like structure.

We predict angles of the protruding convex part against the horizontal line which should be compared with observation.

The relative phase of the amplitudes of this unstable waves for temperature, water-vapor, cloud-water and velocity show that this unstable wave transports heat, water-vapor and cloud-water from inside of the cloud to the surrounding clear air and dilutes the cloud. Therefore we regard this unstable wave as nothing but the mechanism of entrainment or mixing.

The strategy of this study is as follows: 1) First we divide the cloud region into subregions and obtain the mean value in each subregion. Then we obtain linear perturbation equations which describe the motion of the fine structure in the subregion. The term "local" in the title means that we study the stability of the cloud system for each subregion. At this stage we obtain only analytic and algebraic equations.

2) By performing numerical experiment we produce a typical convective cloud. Then by regarding the grid point values of the physical variables as the averaged values at the subregion whose scale is equal to that of the grid size, we substitute them into the coefficients of the perturbation equations. Then we solve the eigenvalue equation and corresponding eigenfunctions numerically.

3) We obtain the maximum eigenvalue and its eigenfunction for each subregion. We assume that this mode is realized in nature and predict the angle of the protruding convex part against the horizontal line.

4) By making a simple model for the absolute value of the amplitude, we obtain a flux of temperature, wate-vapor and cloud water from inside of the cloud to the surrounding clear air.

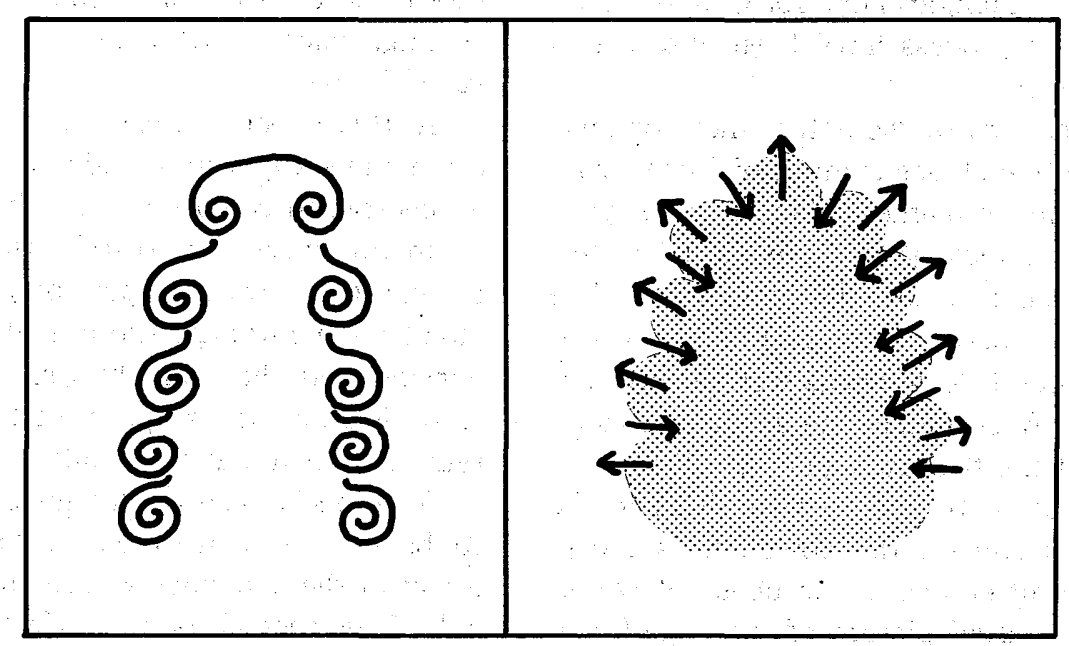

Fig. 1 Left picture shows the entrainment process in the experimental thermal or the plume which is highly nonlinear. Right one shows the entrainment process in the convective cloud. 


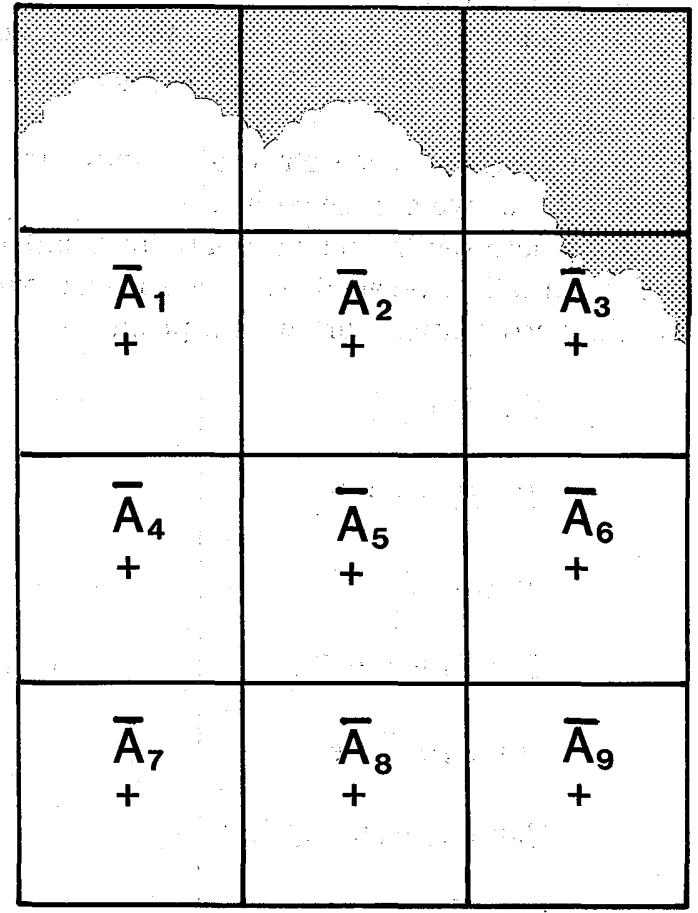

Fig. 2 The subregions of the cloudy air at each of which we solve the instability theory.

\section{Formulation of the problem}

\subsection{Separation of perturbation field}

In order to study the local stability of convective cloud, we divide the cloud region into some subregions whose scale is a few hundred meters. Then we solve the problem at each subregion separately, assuming that there are no correlations in the neighbouring subregions. Now we divide the physical variables into three parts. The first part is the hydrostatic part. The second part is a spatially averaged value over a subregion. When the gradient of the average value is required, it is calculated taking difference of the values in the next-neighbouring subregions. Namely,

$$
\left(\frac{\overline{\partial A}}{\partial x}\right)_{5}=\frac{\overline{A_{6}}-\overline{A_{4}}}{D x}
$$

where $D x$ is the size of the region in $x$ direction.

Further we assume that these average values are constant in time for about one hundred seconds, which is a good approximation in the real cloud. The stratification of these mean fields are tilted from the horizon as we described before.

Finally we assume that the third part describes the fine structure of the convective cloud whose wavelength is supposed to be between several meters and a few tens of meters. We regard this part as a perturbation. The coefficients of these perturbation equations are given by the quantities of hydrostatic and the averaged fields.

\subsection{Configuration of the problem}

In this paper we assume that the averaged field is essentially two-dimensional in each subregion; namely there is a direction in which the mean fields are approximately constant and the mean wind vanishes.*)

We introduce the Cartesian coordinate system, so that the averaged fields are constant in the $y$ direction and the gravity force acts in $z$ direction. Therefore the mean fields depend on $x$ and $z$ coordinates.

In this paper, we take into account the effect of the shear of the mean velocity in the linear approximation form:

$$
\bar{V}_{i}=\bar{V}_{i 0}+\tau_{i j} r_{j}
$$

where $\tau_{i j}$ means the shear tensor of the mean velocity field and the suffixes $i$ and $j$ mean $x$ and $z$ respectively. The repeated use of the same symbol in suffix shows the summation over that suffix (Einstein convention). The coordinates $r_{x}$ and $r_{z}$ are $x$ and $z$ respectively.

\subsection{Boundary conditions}

We assume that fine-scale disturbances in a subregion is independent of those in the neighbouring subregion. This assumption may be justified when the scale of the subregion is large enough compared with the wavelength of the disturbance. Therefore it is possible to consider that the boundary is located infinitely distant. Then our assumption about the bound-

*) But this does not mean that we think only of slab symmetric clouds. It means that the local structure is two-dimensional. 
ary condition is that the amplitude of the disturbance is finite at infinity.

\subsection{Governing equations} tions.

First we write down the fundamental equa-

The equation of motion is

$$
\frac{\partial \vec{V}}{\partial t}=-(\vec{V} \cdot \vec{\nabla}) \vec{V}-\frac{1}{\rho} \vec{\nabla} P-\vec{k} g .
$$

The mass conservation equation is assumed to be anelastic:

$$
\operatorname{div} \cdot(\rho \vec{V})=0 .
$$

In order to include the evaporation and the condensation process into our theory, we assume the following equations.

The first law of thermodynamics is

$$
\frac{d \Theta}{d t}=\frac{L_{v}}{C_{p d}} \frac{\Theta}{T} \beta \frac{Q_{v}-Q_{w s}(T)}{1+\frac{L_{v}}{C_{p d}} \frac{d Q_{w s}(T)}{d T}} .
$$

Here the potential temperature $\Theta$ is approximated to mean the dry potential temperature. The equations of condensation and evaporation are

$$
\frac{d Q_{v}}{d t}=-\beta \frac{Q_{v}-Q_{w s}(T)}{1+\frac{L_{v}}{C_{p d}} \frac{d Q_{w s}(T)}{d T}}
$$

and

$$
\frac{d Q_{c}}{d t}=+\beta \frac{Q_{v}-Q_{w s}(T)}{1+\frac{L_{v}}{C_{p d}} \frac{d Q_{w s}(T)}{d T}} .
$$

The meanings of the symbols are summarized in Appendix A. The unknown parameter $\beta$ gives an inverse of the relaxation time of the oversaturated wet air to approach its saturation level. This is proved in Appendix B. The value of the parameter $\beta$ is of the order of $10 \mathrm{sec}^{-1}$ in a standard cumulus cloud. Estimation of this value will also be given in Appendix B.

Next we will derive the perturbation equations which describe the fine structure of the convective clouds. In this study, the rain water is neglected because we want to simplify the problem in understanding the entrainment and mixing process.

The eddy viscosity terms are also omitted here because we later study inviscid perturbation equations.

Then we divide each physical quantity into three parts as we explained in 2.1.

We denote the hydrostatic part by a suffix $s$, the spatially averaged part by an over bar and the perturbation part with a prime.

$$
\begin{aligned}
& \vec{V}=\overline{\vec{V}}+\vec{v}^{\prime} . \\
& P=P_{s}+\vec{P}+P^{\prime} . \\
& \rho=\rho_{s}+\bar{\rho}+\rho^{\prime} . \\
& T=T_{s}+\bar{T}+T^{\prime} . \\
& \Theta=\Theta_{s}+\bar{\Theta}+\Theta^{\prime} . \\
& Q_{v}=Q_{v s}+\bar{Q}_{v}+Q_{v}{ }^{\prime} . \\
& Q_{c}=\bar{Q}_{c}+Q_{c}{ }^{\prime} \ldots
\end{aligned}
$$

The hydrostatic balance is expressed as follows:

$$
\frac{1}{\rho_{s}} \frac{d P_{s}}{d z}+g=0
$$

The equations for the quantities with a prime in their linearly approximated form are as follows:

$$
\begin{gathered}
\frac{\partial \vec{v}^{\prime}}{\partial t}=-(\overrightarrow{\vec{V}} \cdot \vec{\nabla}) \vec{v}^{\prime}-\left(\vec{v}^{\prime} \cdot \vec{\nabla}\right) \overrightarrow{\vec{V}} \\
-\vec{k}\left(\frac{\rho^{\prime}}{\rho_{s}}\right)-\frac{1}{\rho_{s}} \vec{\nabla} P^{\prime}, \\
\operatorname{div} \cdot\left(\rho_{s} \vec{v}^{\prime}\right)=0, \\
\frac{\partial \Theta^{\prime}}{\partial t}=-\left(\vec{v}^{\prime} \cdot \vec{\nabla}\right)\left(\Theta_{s}+\bar{\Theta}\right)-(\overline{\vec{V}} \cdot \vec{\nabla}) \Theta^{\prime} \\
+\frac{L_{v}\left(\Theta_{s}+\bar{\Theta}\right)}{C_{p d}\left(T_{s}+\bar{T}\right)} \beta \frac{\left(Q_{v}^{\prime}-Q_{w s}\right)}{1+\frac{L_{v}^{\prime}}{C_{p d}}\left(\frac{d Q_{w s}}{d T}\right)_{T=T_{s}+\bar{T}}}
\end{gathered}
$$




$$
\begin{aligned}
\frac{\partial Q_{v}}{\partial t}= & -\left(\vec{v}^{\prime} \cdot \vec{\nabla}\right)\left(Q_{v s}+\bar{Q}_{v}\right)-(\overline{\vec{V}} \cdot \vec{\nabla}) Q_{v}{ }^{\prime} \\
& -\beta \frac{\left(Q_{v}{ }^{\prime}-Q_{w s}{ }^{\prime}\right)}{1+\frac{L_{v}}{C_{p d}}\left(\frac{d Q_{w s}}{d T}\right)_{T=T_{s}+\bar{T}}}, \\
\frac{\partial Q_{c}{ }^{\prime}}{\partial t}= & -\left(\vec{v}^{\prime} \cdot \vec{\nabla}\right) Q_{c}-(\overline{\vec{V}} \cdot \vec{\nabla}) Q_{c}{ }^{\prime} \\
& +\beta \frac{\left(Q_{v}{ }^{\prime}-Q_{w s}{ }^{\prime}\right)}{1+\frac{L_{v}}{C_{p d}}\left(\frac{d Q_{w s}}{d T}\right)_{T=T_{s}+\bar{T}}}
\end{aligned}
$$

where

$$
Q_{w s}^{\prime}=\left(\frac{d Q_{w s}}{d T}\right)_{T=T_{s}+\bar{T}}\left(\frac{P_{d s}+\bar{P}_{d}}{P_{s s}}\right)^{e} \Theta^{\prime}
$$

and

$$
e=R_{d} / C_{p d} .
$$

To obtain Eqs.(2.9) (2.13), it is assumed that

$$
Q_{v s}+\bar{Q}_{v}-Q_{w s}\left(T_{s}+\bar{T}\right) \simeq 0 .
$$

The buoyancy term and $z$-component of the pressure gradient force in Eq.(2.9) are combined to become

$$
\begin{aligned}
& -\vec{k}\left(g \frac{\rho^{\prime}}{\rho_{s}}+\frac{1}{\rho_{s}} \frac{\partial P^{\prime}}{\partial z}\right) \\
& =-\vec{k}\left[g \frac{\rho^{\prime}}{\rho_{s}}+\frac{\partial}{\partial z}\left(\frac{P^{\prime}}{\rho_{s}}\right)+\frac{P^{\prime}}{\rho_{s}{ }^{2}} \frac{d \rho_{s}}{d z}\right] \\
& \simeq-\vec{k} \frac{\partial}{\partial z}\left(\frac{P^{\prime}}{\rho_{s}}\right) \\
& +\vec{k} g\left[\frac{\Theta^{\prime}}{\Theta_{s}}+\left(\frac{m_{d}}{m_{v}}-1\right) Q_{v}{ }^{\prime}-Q_{c}{ }^{\prime}\right] \\
& +\frac{P^{\prime}}{P_{s}} \vec{k} R_{d} T_{s}\left[1+\left(\frac{m_{d}}{m_{v}}-1\right) Q_{v s}\right] \\
& \quad \times\left[\frac{1}{\Theta_{s}} \frac{d \Theta_{s}}{d z}+\left(\frac{m_{d}}{m_{v}}-1\right) \frac{d Q_{v s}}{d z}\right] .
\end{aligned}
$$

If we assume that $\Theta^{\prime} / \Theta_{s}, Q_{v}{ }^{\prime}, Q_{c}{ }^{\prime}, P^{\prime} / P_{s}$ are the same order and know the fact that

$$
\begin{aligned}
& R_{d} T_{s} \simeq 10^{5} \mathrm{Joul} / \mathrm{kg}, \\
& \frac{1}{\Theta_{s}} \frac{d \Theta_{s}}{d z} \simeq 10^{-5} \mathrm{~m}^{-1},
\end{aligned}
$$

$$
\frac{d Q_{v s}}{d z}\left(\frac{m_{d}}{m_{v}}-1\right) \simeq 10^{-6} \mathrm{~m}^{-1},
$$

we can conclude that the second term in Eq.(2.16) is about 10 times as large as the third term. Therefore, we neglect the third term in Eq.(2.16) hereafter.

Further we assume that the scale height is very large compared with the wavelength of the disturbance $\lambda$

$$
\left|\frac{1}{\rho_{s}} \frac{d \rho_{s}}{d z}\right| \ll \frac{1}{\lambda} .
$$

Therefore we assume that

$$
\operatorname{div} \cdot\left(\vec{v}^{\prime}\right)=0 \text {. }
$$

The final form of the equation for perturbation velocity becomes

$$
\begin{aligned}
\frac{\partial \vec{v}^{\prime}}{\partial t}= & -(\overrightarrow{\vec{V}} \cdot \vec{\nabla}) \vec{v}^{\prime}-\left(\vec{v}^{\prime} \cdot \vec{\nabla}\right) \overline{\vec{V}} \\
& +\vec{k} g\left[\frac{\Theta^{\prime}}{\Theta_{s}+\bar{\Theta}}+\left(\frac{m_{d}}{m_{v}}-1\right) Q_{v^{\prime}}-Q_{c}{ }^{\prime}\right] \\
& -\vec{\nabla}\left(\frac{P^{\prime}}{\rho_{s}}\right) .
\end{aligned}
$$

\subsection{Numerical model}

In order to get the quantitative results from our theory, the mean wind and its shear, the mean stratification of the temperature, watervapor and cloud-water inside of the cloud must be given. We obtain these necessary data from the results of numerical simulation. Namely we produce a typical convective cloud by numerical simulation and regard the grid point data as the averaged value over the region whose scale is just equal to the grid size. In our model, the grid size is 200 meters.

The scheme of our numerical model is as follows:

1) Anelastic and non-hydrostatic system.

2) Two-dimensional Cartesian coordinate system with horizontal scale $25.6 \mathrm{~km}$ and vertical scale $12.8 \mathrm{~km}$.

3) The grid size is 200 meters both horizontally and vertically.

4) The time step is taken to be 10 seconds and 
a forward difference scheme is employed.

5) The vertical boundary condition is closed and free slip. The horizontal boundary condition is periodic.

6) The advective terms of the momentum equations are written in the upstream method. 7) The advective terms for the scalar quantities are written in the form of the modified upstream method (Soong and Ogura, 1973) and the staggered grids are used.

8) The perturbed pressure is neglected when we use Teten's formula in order to relate temperature and saturated water vapor pressure. (Wilhelmson and Ogura, 1972)

9) The auto-conversion term is parameterized by Kessler's formula.

10) The size distribution of the rain drop is assumed to be the Marshal-Palmer type.

11) Initially there are no clouds and a cloud is triggered by giving the following sensible heat flux :

$$
J=f_{0} \exp \left(-x^{2} / \Sigma^{2}\right) \exp \left(-z / \alpha_{0}\right),
$$

where

$$
\begin{aligned}
& f_{0}=700 \mathrm{watt} / \mathrm{m}^{2}, \\
& \Sigma=1000 \mathrm{~m}, \\
& \alpha_{0}=200 \mathrm{~m}
\end{aligned}
$$

and heating is continued during the first 15 minutes.

12) The initial stratification is shown in Fig. 3.

13) The eddy terms are composed of two terms, one of which is a constant diffusion coefficient term with the value $25 \mathrm{~m}^{2} / \mathrm{sec}$. The other term is not zero only inside of the cloud and parameterized by using the mode of the largest growth-rate in our theory at each grid point.

\section{Method of solution}

In this section we obtain an algebraic equation for the eigenvalue.

3.1 A coordinate system moving with velocity field

When we observe the movement of the

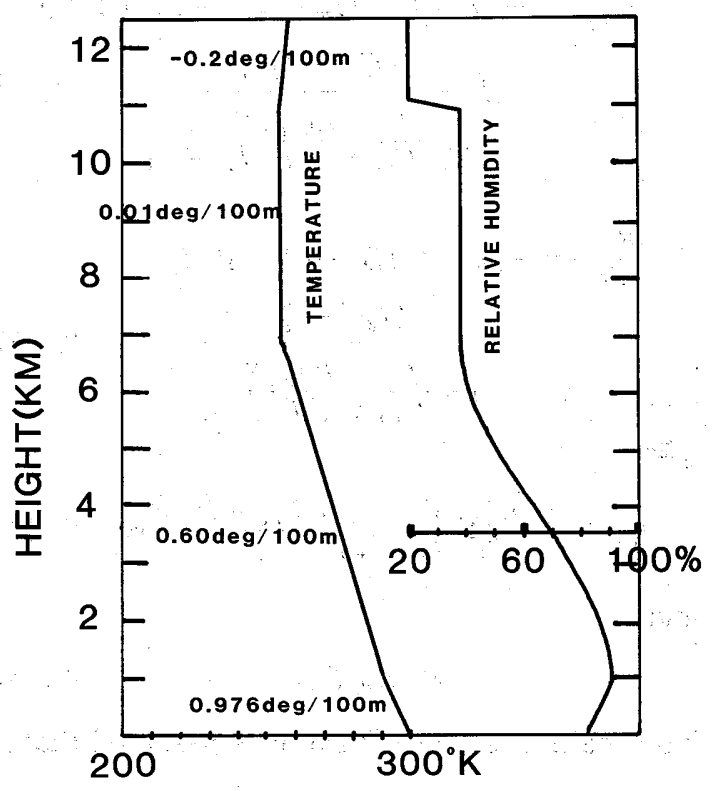

Fig. 3 Initial stratification of the numerical model through which we : obtain cloud physical data inside of the cloud.

boundary of convective clouds, all the uneven structures show translation and transformation because of wind and its shear. To take the effect of the wind into account, we employ the following coordinate system which moves with the mean wind:

$$
r_{i}{ }^{\prime}=r_{i}-\left(\bar{V}_{i 0}+\tau_{i j} r_{j}\right) t
$$

and

$$
t^{\prime}=t
$$

The relationship between the derivative operators in the old and new coordinate systems are given as

$$
\frac{\partial}{\partial r_{i}}=\left(\delta_{i j}-\tau_{j i} t^{\prime}\right) \frac{\partial}{\partial r_{j}^{\prime}}
$$

and

$$
\frac{\partial}{\partial t}=\frac{\partial}{\partial t^{\prime}}-\bar{V}_{i} \frac{\partial}{\partial r_{i}^{\prime}}
$$

where the $\delta_{i j^{\prime}}$ means the Kronecker delta.

Then the local time derivative term with the advection term by the mean velocity becomes 


$$
\frac{\partial}{\partial t}+\bar{V}_{i} \frac{\partial}{\partial r_{i}}=\frac{\partial}{\partial t^{\prime}}-\bar{V}_{i} \tau_{j i} t^{\prime} \frac{\partial}{\partial r_{j}^{\prime}}
$$

In a typical convective cloud, the shear of the mean wind is of the order of $10^{-2} \mathrm{sec}^{-1}$. Therefore, so far as

$$
\left|\tau_{i j} t\right| \ll 1 \text {, }
$$

the effect of shear is negligible in Eq.(3.3) and we put

$$
\frac{\partial}{\partial r_{i}}=\frac{\partial}{\partial r_{i}^{\prime}} .
$$

We define the term "growing stage" by the time duration in which Eq.(3.6) remains a good approximation after the perturbation is switched on. The duration is of the order of tens of seconds.

Next we assume

$$
\frac{\partial}{\partial t^{\prime}}-\bar{V}_{i} \tau_{j i} t^{\prime} \frac{\partial}{\partial r_{j}^{\prime}}=\frac{\partial}{\partial t^{\prime}}-\bar{V}_{i 0} \tau_{j i} t^{\prime} \frac{\partial}{\partial r_{j}^{\prime}} .
$$

This assumption means that we put

$$
\left|\bar{V}_{i 0}\right| \gg\left|\tau_{i j} r_{j}\right|
$$

in each subregion. ${ }^{*}$

\subsection{Stream function for the perturbation veloci ty}

We assume that the perturbation fields are

*) Actually this is a rather severe restriction. Eq. (3.8) can be violated at places where the mean velocity is small but the shear is large. Such places are likely to be located in the central region of the cloud top and at two centers of the dipole-like circulation of the mean velocity. Appendix $D$ is devoted to the discussion about this restriction, while Appendix $\mathrm{E}$ deals with the effect of the neglected term. We think that this term will contribute to changing the wavelength place by place and is not significant for the analysis of the instability.

**) By solving the more general equation in which the perturbation is allowed to be three-dimensional, we confirmed that perturbation whose growth-rate is maximum has its velocity in the $x-z$ plane. Because we are concerned only with the most unstable mode, it is enough to study the two-dimensional case. also uniform in the $y$-direction, and the perturbation velocity is confined to the $x-z$ plane.**) Then a stream function $\Psi^{\prime}(x, z)$ for the velocity field may be introduced:

$$
v_{x}{ }^{\prime}=-\frac{\partial \Psi^{\prime}}{\partial z^{\prime}} \quad \text { and } \quad v_{z}{ }^{\prime}=\frac{\partial \Psi^{\prime}}{\partial z^{\prime}} .
$$

Taking the $y$-component of the curl of Eq.(2.18) and by using Eqs.(3.5), (3.7), (3.9), we obtain

$$
\begin{aligned}
& \left(\frac{\partial}{\partial t^{\prime}}-\bar{V}_{i 0} \tau_{j i} t^{\prime} \frac{\partial}{\partial r_{j}^{\prime}}+\operatorname{div} \cdot \overline{\vec{V}}\right)\left(-\vec{\nabla}^{2} \Psi^{\prime}\right) \\
=- & (\vec{j} \cdot \vec{k} \times \vec{\nabla}) g \\
& \quad \times\left[\frac{\Theta^{\prime}}{\Theta_{s}+\bar{\Theta}}+\left(\frac{m_{d}}{m_{v}}-1\right) Q_{v^{\prime}}-Q_{c}{ }^{\prime}\right] \cdot(3.10)
\end{aligned}
$$

\subsection{Plane wave solution}

If we assume that all the averaged values and their gradients are constant in space, Eqs.(2.11), (2.12), (2.13) and (3.10) become a set of simultaneous linear differential equations with constant coefficients in space.

We will consider a plane wave solution of the form

$$
\exp \left(-i \vec{p} \cdot \vec{r}^{\prime}\right)
$$

where $\vec{r}^{\prime}$ is a coordinate vector defined in Eq.(3.1), $\vec{p}$ a wavenumber vector in the $x z$ plane. This solution automatically satisfies the boundary condition discussed in section 2 .

Now let us introduce three orthonormal vectors $\vec{i}_{1}, \vec{i}_{2}$ and $\vec{i}_{3}$ defined by

$$
\begin{aligned}
& \vec{i}_{1}=\frac{\vec{k} \times \vec{p}}{|\vec{k} \times \vec{p}|}, \\
& \vec{i}_{2}=\frac{\vec{p} \times(\vec{k} \times \vec{p})}{|\vec{p}||\vec{k} \times \vec{p}|}, \\
& \vec{i}_{3}=\frac{\vec{p}}{|\vec{p}|} .
\end{aligned}
$$

The vector $\vec{i}_{1}$ is no other than $\vec{j}$.

Then we replace every space derivative in the following way:

$$
\vec{\nabla}^{\prime}=-i|\vec{p}| \vec{i}_{3}
$$

Eq.(3.10) in then rewritten as 


$$
\begin{aligned}
& \left(\frac{\partial}{\partial t^{\prime}}+\bar{V}_{i 0} \tau_{j i} t^{\prime} i(\vec{p})_{j}+\operatorname{div} \cdot \overrightarrow{\vec{V}}\right)\left(+\vec{p}^{2} \Psi^{\prime}\right) \\
= & i|\vec{k} \times \vec{p}| g\left[\frac{\Theta^{\prime}}{\Theta_{s}+\bar{\Theta}}+\left(\frac{m_{d}}{m_{v}}-1\right) Q_{v}^{\prime}-Q_{c}^{\prime}\right]
\end{aligned}
$$

In each equation, the time dependence of the coefficients only comes through the term

$$
\bar{V}_{i 0} \tau_{j i} t^{\prime} i(\vec{p})_{j} .
$$

This term can be easily eliminated by the following transformation for the perturbation function:

$$
A^{\prime}=B^{\prime} \exp \left[-\frac{1}{2} \bar{V}_{i 0} \tau_{j i} t^{\prime 2} i(\vec{p})_{j}\right]
$$

where the symbol $A^{\prime}$ denotes any of the perturbation quantities. The factor contributes only to the change of phase of the function and not to the change of amplitude with time.

The perturbation equations are homogeneous and we can factor this out from all the perturbation functions.

The new perturbation equation becomes, for example

$$
\begin{aligned}
& \left(\frac{\partial}{\partial t^{\prime}}+\operatorname{div} \cdot \overrightarrow{\vec{V}}\right)\left(+\vec{p}^{2} \Psi^{\prime}\right) \\
= & i|\vec{k} \times \vec{p}| g\left[\frac{\Theta^{\prime}}{\Theta_{s}+\bar{\Theta}}+\left(\frac{m_{d}}{m_{v}}-1\right) Q_{v}^{\prime}-Q_{c}^{\prime}\right]
\end{aligned}
$$

The equations for the scalar quantities are modified in a similar manner.

Since all the coefficients are independent of time, we can assume the following time dependence for the perturbation function:

$$
\exp \left(\sigma t^{\prime}\right)
$$

$$
\chi=\frac{1+\frac{m_{d}}{m_{v}}\left(\frac{d Q_{w s}}{d T}\right)_{T=T s+\bar{T}}\left(T_{s}+\bar{T}\right)}{1+\frac{L_{v}}{C_{p d}}\left(\frac{d Q_{w s}}{d T}\right)_{T=T_{s}+\bar{T}}}=\frac{1+\frac{m_{d} L_{v} Q_{w s}}{m_{v}\left(T_{s}+\bar{T}\right) R_{v}}}{1+\frac{L_{v}^{2} Q_{w s}}{C_{p d} R_{v}\left(T_{s}+\bar{T}\right)^{2}}}
$$

and 


$$
\lambda=\frac{\frac{L_{v}}{C_{p d}\left(T_{s}+\bar{T}\right)}-\frac{m_{d}}{m_{v}}}{1+\frac{L_{v}}{C_{p d}}\left(\frac{d Q_{w s}}{d T}\right)_{T=T_{s}+\bar{T}}}+\frac{m_{d}}{m_{v}}-1=\frac{\frac{L_{v}}{C_{p d}\left(T_{s}+\bar{T}\right)}-\frac{m_{d}}{m_{v}}}{1+\frac{L_{v}^{2} Q_{w s}}{C_{p d} R_{v}\left(T_{s}+\bar{T}\right)^{2}}}+\frac{m_{d}}{m_{v}}-1
$$

If we note that the value of $\beta$ is of the order of $10 \mathrm{sec}^{-1}$ and $a_{0}, a_{1}, a_{2}, b_{1}$ and $b_{2}$ are of the order of $10^{-2} \mathrm{sec}^{-1}$, we can obtain the following approximate root for Eq.(3.8): ${ }^{*}$

$$
\left.\begin{array}{l}
\sigma_{1} \approx-\beta+a_{0} \\
\sigma_{2} \approx a_{1}+b_{1} / \beta \\
\sigma_{3} \approx a_{2}+b_{2} / \beta
\end{array}\right\}
$$

The relation between roots and coefficients gives the following equations:

$$
\left.\begin{array}{c}
a_{0}+a_{1}+a_{2}=a_{1}+a_{2}=-\operatorname{div} \cdot \overrightarrow{\vec{V}} \\
\therefore \quad a_{1} a_{2}=g \frac{|\vec{k} \times \vec{p}|}{|\vec{p}|} B_{, 2},
\end{array}\right\}
$$

The divergence term in Eq.(3.26) is unually very small $\left(10^{-4} \sim 10^{-3} \mathrm{sec}^{-1}\right)$ and we can neglect them. Then the solutions for this equation are easily solved to give

$$
a_{0}=0 \text {, }
$$

$$
\begin{aligned}
& a_{1}=+\sqrt{-g \frac{|\vec{k} \times \vec{p}|}{|\vec{p}|} B_{, 2},} \\
& a_{2}=-\sqrt{-g \frac{|\vec{k} \times \vec{p}|}{|\vec{p}|} B_{, 2}},
\end{aligned}
$$

$$
b_{1}=b_{2}=\frac{1}{2} g \frac{|\vec{k} \times \vec{p}|}{|\vec{p}|} \delta\left[\vec{i}_{2} \cdot \vec{\nabla}\left(Q_{v s}+\bar{Q}_{v}\right)-\left(\frac{d Q_{w s}}{d T}\right)_{T=T_{s}+\bar{T}}\left(T_{s}+\bar{T}\right) \frac{1}{\Theta_{s}+\bar{\Theta}} \vec{\nabla}\left(\Theta_{s}+\bar{\Theta}\right)\right]
$$

The order of the $b_{1}$ and $b_{2}$ are $10^{-4} \sim 10^{-3}$ $\mathrm{sec}^{-2}$. In the last two equations of Eq.(3.25), the the second term in the righthand side is of the order of $10^{-5} \sim 10^{-4} \mathrm{sec}^{-1}$, and $a_{1}, a_{2}$ of the

*) In a case where the cloud-water content is very small, $\beta$ is aslo small and this approximation may not be valid. Thus, we need to solve Eq. (3.18) directly and the growth rate starts to depend on $\beta$ explicitly. order of $10^{-2} \mathrm{sec}^{-1}$. Therefore the $\sigma_{2}$ and $\sigma_{3}$ are given accurately enough by $a_{1}$ and $a_{2}$.

As we will see in the next section, our theory gives only the direction of the wavenumber vector $\vec{p}$ to give the maximum growth rate but not the magnitude. Perturbations do not depend on the magnitude of the wavenumber vector.

\subsection{Expression for the amplitudes of the per- turbations}

In this subsection, we give the expression for the amplitudes of the perturbation quantities:

$$
\vec{v}^{\prime}=\operatorname{Re}\left(-i|\vec{p}| \vec{i}_{2} \Psi_{1}^{\prime}\right),
$$


$\Theta^{\prime}=\frac{\Theta_{s}+\bar{\Theta}}{\sigma\left(1+\frac{L_{v}^{2} Q_{w s}}{C_{p d} R_{v}\left(T_{s}+\bar{T}\right)^{2}}\right)}\left[\vec{v}^{\prime} \cdot \frac{-1}{\Theta_{s}+\bar{\Theta}} \vec{\nabla}\left(\Theta_{s}+\bar{\Theta}\right)-\frac{L_{v}}{C_{p d}\left(T_{s}+\bar{T}\right)} \vec{\nabla}\left(Q_{v s}+\bar{Q}_{v}\right)\right]$

$Q_{v}{ }^{\prime}=\frac{\frac{L_{v} Q_{w s}}{R_{v}\left(T_{s}+\bar{T}\right)}}{\sigma\left(1+\frac{L_{v}{ }^{2} Q_{w s}}{C_{p d} R_{v}\left(T_{s}+\bar{T}\right)^{2}}\right)}\left[\vec{v}^{\prime} \cdot \frac{-1}{\Theta_{s}+\bar{\Theta}} \vec{\nabla}\left(\Theta_{s}+\bar{\Theta}\right)-\frac{L_{v}}{C_{p d}\left(T_{s}+\bar{T}\right)} \vec{\nabla}\left(Q_{v s}+\bar{Q}_{v}\right)\right]$,

$$
Q_{c}^{\prime}=\frac{1}{\sigma}\left[\vec{v}^{\prime} \cdot-\vec{\nabla} \bar{Q}_{c}-\frac{\vec{\nabla}\left(Q_{v s}+\bar{Q}_{v}\right)-\frac{L_{v} Q_{w s}}{R_{v}\left(T_{s}+\bar{T}\right)\left(\Theta_{s}+\bar{\Theta}\right)} \vec{\nabla}\left(\Theta_{s}+\bar{\Theta}\right)}{1+\frac{L_{v}{ }^{2} Q_{w s}}{C_{p d} R_{v}\left(T_{s}+\bar{T}\right)^{2}}}\right]
$$

and

$$
\Psi_{1}{ }^{\prime}=\Psi_{10} \exp \left(\sigma t^{\prime}-i \vec{p} \cdot \vec{r}^{\prime}\right),
$$

where Re means the real part.

3.6 Equations for the flux

Using the expression in the previous sub-

section, we can calculate the magnitudes of mean heat flux, water-vapour flux and cloudwater flux vectors along the direction $\vec{i}_{2}$.

The word "mean" means an averaged value over one wavelength in the direction of the wavenumber vector $\vec{p}$.

heat flux:

$$
\begin{aligned}
& \overline{\Theta^{\prime}\left(\vec{i}_{2} \cdot \vec{v}^{\prime}\right)}=\frac{1}{\sigma^{\prime}}\left|\vec{v}^{\prime}\right|^{2} \\
& \quad \times \frac{\Theta_{s}+\bar{\Theta}}{L_{v}^{2} Q_{w s}}\left[\vec{i}_{2} \cdot-\frac{1}{\Theta_{s}+\bar{\Theta}} \vec{\nabla}\left(\Theta_{s}+\bar{\Theta}\right)-\frac{L_{v}}{C_{p d}\left(T_{s}+\bar{T}\right)} \vec{V}\left(Q_{v s}+\bar{Q}_{v}\right)\right]
\end{aligned}
$$

water-vapour flux

$$
\begin{aligned}
\overline{Q_{v}{ }^{\prime}\left(\vec{i}_{2} \cdot \vec{v}^{\prime}\right)} & =\frac{1}{\sigma}\left|\overline{\vec{v}}^{\prime}\right|^{2} \\
& \times \frac{\frac{L_{v} Q_{w s}}{R_{v}\left(T_{s}+\bar{T}\right)}}{1+\frac{L_{v}{ }^{2} Q_{w s}}{C_{p d} R_{v}\left(T_{s}+\bar{T}\right)^{2}}}\left[\vec{i}_{2}-\frac{1}{\Theta_{s}+\bar{\Theta}} \vec{\nabla}\left(\Theta_{s}+\bar{\Theta}\right)-\frac{L_{v}}{C_{p d}\left(T_{s}+\bar{T}\right)} \vec{\nabla}\left(Q_{v s}+\bar{Q}_{v}\right)\right]
\end{aligned}
$$


cloud-water flux

$$
\overline{Q_{c}^{\prime}\left(\vec{i}_{2} \cdot \vec{v}^{\prime}\right)}=\frac{1}{\sigma}\left|\overrightarrow{\vec{v}}^{\prime}\right|^{2}\left[\vec{i}_{2} \cdot-\vec{\nabla} \vec{Q}_{c}-\frac{\vec{\nabla}\left(Q_{v s}+\bar{Q}_{v}\right)-\frac{L_{v} Q_{w s}}{R_{v}\left(T_{s}+\bar{T}\right)\left(\Theta_{s}+\bar{\Theta}\right)} \vec{\nabla}\left(\Theta_{s}+\bar{\Theta}\right)}{1+\frac{L_{v}^{2} Q_{w s}}{C_{p d} R_{v}\left(T_{s}+\bar{T}\right)^{2}}}\right]
$$

\section{Physical mechanism of the instability}

In this section, we will make clear the physical meaning of the instability studied in the former section.

The function inside of the root in Eq.(3.28) is rewritten as

$$
g \frac{|\vec{k} \times \vec{p}|}{|\vec{p}|} B_{, 2}=-g\left(\vec{i}_{2} \cdot \vec{k}\right)\left(\vec{i}_{2} \cdot-\vec{B}\right),
$$

where use is made of Eqs.(3.12) and (3.21).

Calling back the fact that the direction of $\vec{i}_{2}$ is parallel or antiparallel to the vector $\vec{v}^{\prime}$, Eq.(4.1) shows that the maximum value of the growth-rate is obtained when the direction of the perturbation velocity vector $\vec{v}^{\prime}$ is right at the middle of the two vectors $\vec{k}$ and $-\vec{B}$ or $-\vec{k}$ and $\vec{B}$.

In Appendix C, we will show that the vector $-\vec{B}$ has the meaning that if a parcel in a cloud, where the water vapour content is saturate, experiences an infinitesimally small displacement $\Delta \vec{r}$, then it acquires buoyancy of

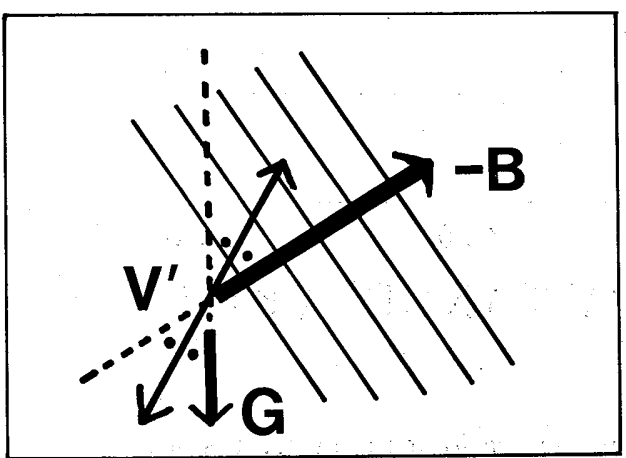

Fig. 4 The predicted direction of the velocity of the unstable wave which is just the middle of the two directions, that of the gravity and the vector $\boldsymbol{B}$.

$$
\Delta \vec{F}_{B}=g \vec{k}(\Delta \vec{r} \cdot-\vec{B}) .
$$

Namely a parcel will acquire the maximum buoyancy $g|\vec{B} \| \Delta \vec{r}|$ if it moves parallel to the vector $-\vec{B}$.

Though possible forces which act on this parcel are buoyancy (vertical) and pressure gradient force whose direction is not vertical in our tilted stratification, the true dynamics shows that the actual motion of the air occurs amid the two directions $\vec{k}$ and $-\vec{B}$.

A simple interpretation by parcel method is as follows:

If possible force on a parcel is only $\Delta \vec{F}_{B}$ in Eq.(4.2), then Newton's equation for this parcel with mass $m$ is as follows:

$$
m \Delta \ddot{\vec{r}}=g \vec{k}(\Delta \vec{r} \cdot-\vec{B}) .
$$

If we denote a unit vector along the direction $\Delta \vec{r}$ by $\vec{r}$, Eq.(4.3) along $\hat{r}$ becomes

$$
m \Delta \ddot{r}=g(\vec{k} \cdot \hat{r})(\hat{r} \cdot-\vec{B}) \Delta r,
$$

Where $\Delta r$ means $(\Delta r \cdot \hat{r})$.

This equation has an exponentially growing solution with growth-rate $\sigma$ if

$$
\sigma^{2}=g(\vec{k} \cdot \hat{r})(\hat{r} \cdot-\vec{B}) / m>0 .
$$

The maximum value of the growth-rate is obtained when the direction of the perturbation velocity vector $\Delta \vec{r}$ is right at the middle of the two vectors $\vec{k}$ and $-\vec{B}$ or $-\vec{k}$ and $\vec{B}$.

Finally we should remark that when the parameter $\beta$ is small, it will explicitly enter into the eigenvalue and the physical interpretation will not be so simple.

\section{Results and discussions}

\subsection{Distribution of the most unstable directions and growth-rate}

Fig. 5 shows the distribution of the cloudwater mixing-ratio 30 minutes after the initial 


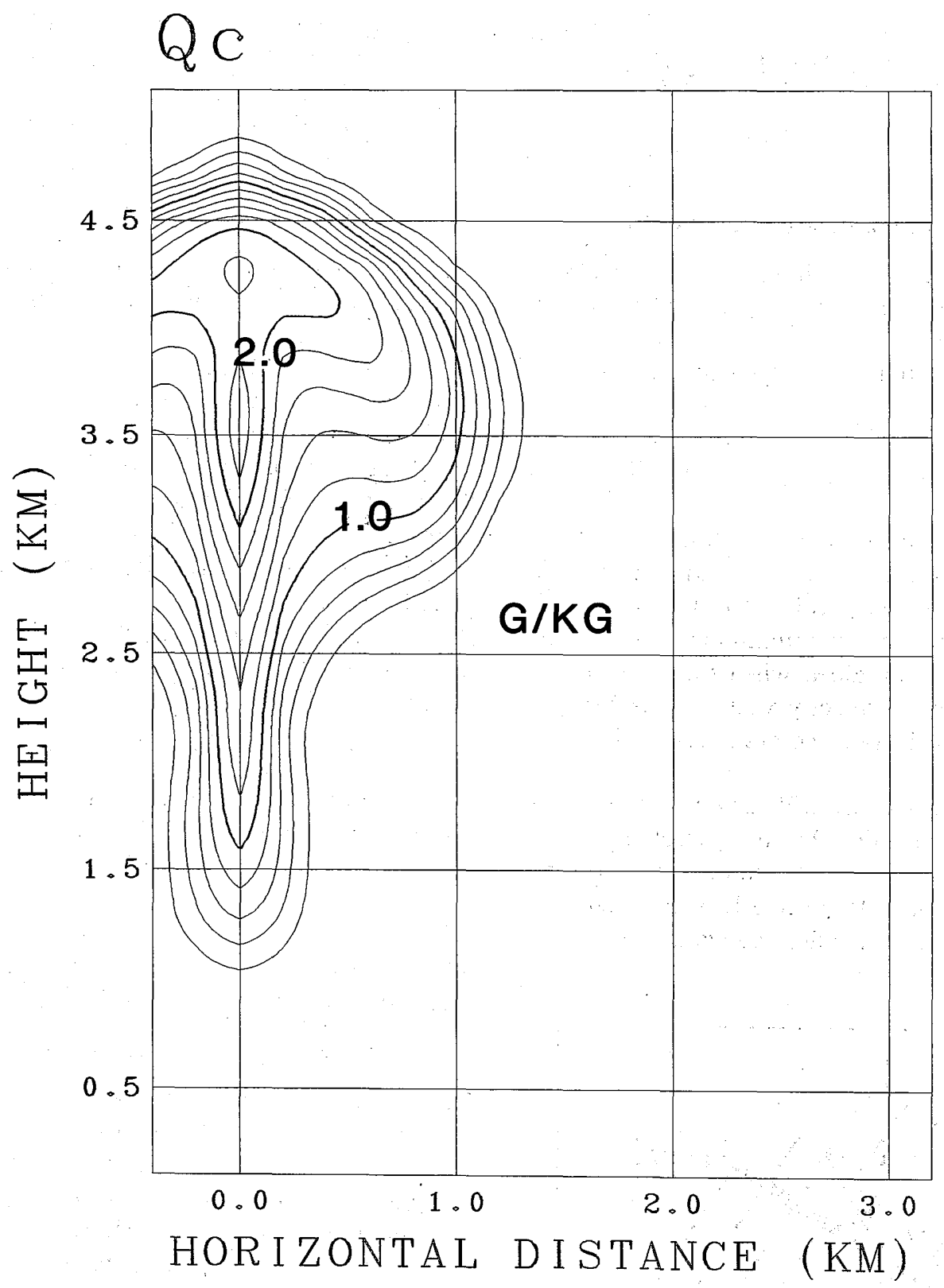

Fig. 5 The distribution of the cloud-water mixing ratio at the matured stage. 


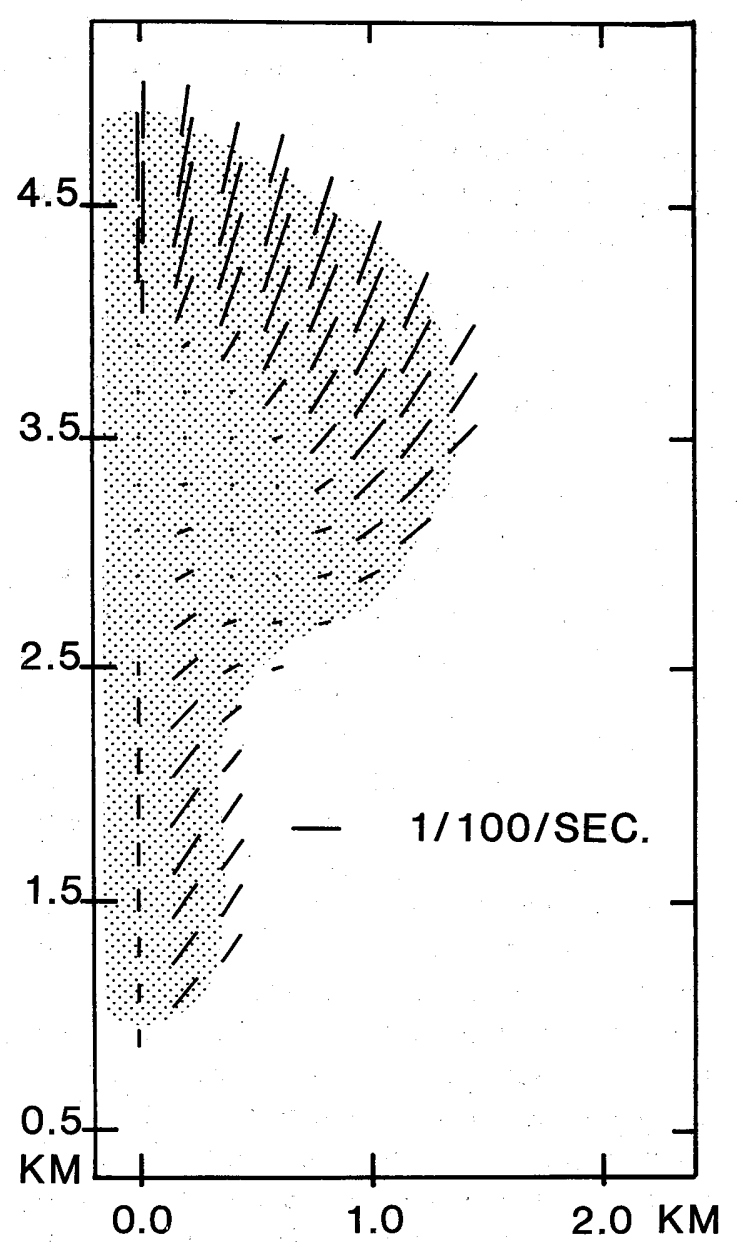

Fig. 6 The distribution of the growth-rate (proportional to the length) and the direction of the velocity associated with the most unstable mode. The shaded region means the cloudy air $\left(Q_{c} \geqq 0.1 \mathrm{~g} / \mathrm{kg}\right)$.

state. This cloud is at the matured stage and the maximum vertical velocity is 13.4 meter/ sec.

Fig. 6 shows the direction of the perturbation velocity of the most unstable mode by an arrow and the magnitude of the growth-rate by the length of the arrow at each grid point. The direction of the velocity of the most unstable mode is nearly vertical at the cloud top and its angle from the horizontal plane becomes smaller as the position of the grid point becomes lower.

We also notice that the growth-rate is larg- est at the cloud top and becomes smaller as the position becomes lower. This result suggests that the instability is strongest at the cloud top. Moreover, in the central part of the cloud where the mean velocity is very large, the growth-rate is very small. This result compares favorably with the observational result that disturbance is weak in the central part of clouds (Paluch and Baumgardener, 1988; Warner, 1969). This is also supported by experiment of flight in clouds in which the airplane rolls and pitches heavily near the boundary but not inside of the cloud (Ichimura, private communication 1988).

\subsection{Relative phases between eigenfunctions}

As we will see in Figs. $8 \sim 10$, the fluxes of the potential temperature, water-vapor and cloud-water expressed in Eqs.(3.36) (3.38) are positive at almost every part of the cloud. ${ }^{*}$ It determines the phase difference between eigenfunctions and also the pattern of the wave stimulated by this instability. Warm, wet and cloudy air moves outward, while cold, dry and less cloudy air moves inward. Thus the instability wave dilutes the cloudy air. This is no other than the entrainment or mixing process.

\subsection{Parameterization of the eddy terms}

Eqs.(3.36) (3.38) suggest a new method of parameterization of the eddy viscosity coefficients. Our idea is that, at each grid point, the eddy transport is done by the mode of the maximum growth-rate. Since our theory is linear, however, the absolute value of the amplitudes cannot be determined. Thus we propose a model for this amplitude in the following way:

$$
\bar{v}^{\prime 2}=(100 \times \sigma)^{n},
$$

where the parameter $\mathrm{n}$ is is still undetermined. By this parameterization, the dependence on $\sigma$ of various quantities are as follows:

*) In order to obtain Figs. $8 \sim 10$, we assumed Eq. (5.1), which we will discuss in 5.3. But the sign of the flux does not depend on the assumption (5.1). 


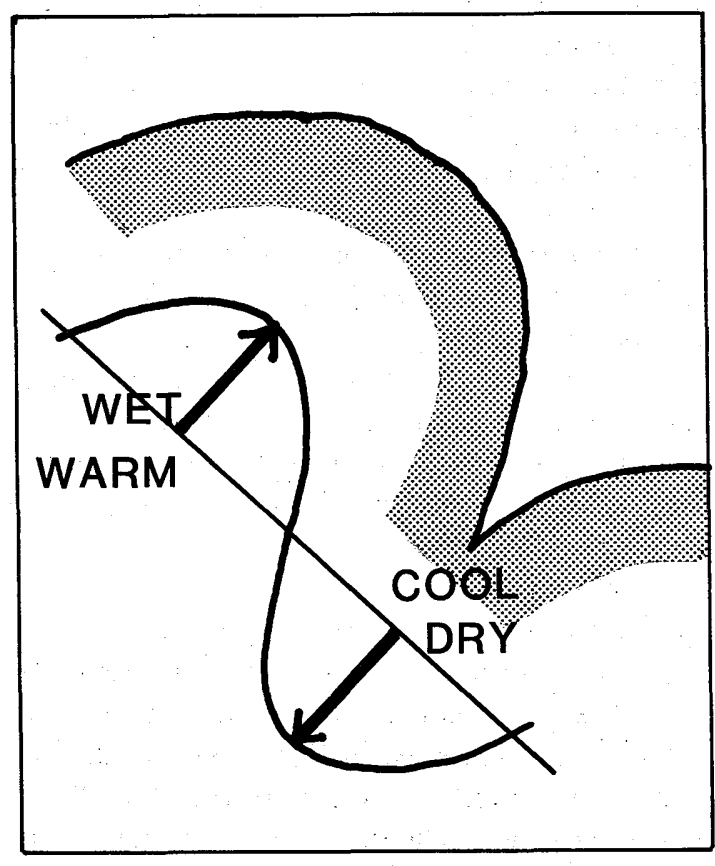

Fig. 7 The phase of the unstable wave. The arrow means the velocity associated with the unstable wave.

fluxes defined in $(3.36) \sim(3.38) \propto \sigma^{n-1}$,

$$
\begin{gathered}
v^{\prime} \propto \sigma^{n / 2}, \\
\Theta^{\prime}, Q_{v}{ }^{\prime} Q_{c}^{\prime} \propto \sigma^{n / 2-1} .
\end{gathered}
$$

At least the flux must vanish when $\sigma=0$. Therefore the parameter $n$ must satisfy

$$
n>1 \text {. }
$$

Temporarily we simply assume that $n=2$. This should be explicitly determined by studying the nonlinear theory or observation. For the larger $n$, the dependence of the flux on $\sigma$ becomes stronger. The multiplicative number 100 is chosen in order to adjust the velocity of the unstable wave to be a few meters per second as the result of the observational study shows.

\subsection{The distribution of fluxes}

In Figs. $8 \sim 10$, we show the distribution of the fluxes of the potential temperature, water- vapor and cloud-water expressed in Eqs.(3.36) $\sim(3.38)$, with the assumption (5.1) and $n=2$. Almost in every part of the cloud, they are positive and strongest at the top part of the cloud. This result supports the hypothesis that the entrainment or mixing is most active at the cloud top (Reuter and Yau, 1987).

If we assume larger $n$, the relative strength of the entrainment or mixing at the cloud top becomes larger.

\subsection{On the uneven structure of the surface of the convective cloud}

Our instability theory is valid only inside of the clouds where the air is saturated and enough cloud-water exists. The instability wave will produce heterogeneousness in the physical variables when it is superposed on the mean value of the subregion. Especially the heterogeneousness of the cloud-water content is important. We apply our theory to the outermost subregion where the mean value of the cloud-water content is small. Then by this unstable wave, the thin and thick wavy distribution of the cloud-water content will appear. Now we assume that this structure reflects the uneven structure of the surface of the cloud. Namely the thick part corresponds to the convex part and the thin part to the concave part. We showed in 5.2 that at the thick part, the velocity of the unstable wave is outward and at the thin part inward. Therefore the convex part protrudes and the concave part sinks.

Then we can see the distribution of the angles of the uneven structure in the case of the convective cloud in its growing stage in Fig. 6 by analyzing the direction of the most unstable mode (thick line) at the peripheral part of the cloud. Even at the side part of the cloud, the convex part may grow to several tens of degrees.with the horizontal line, which we often see through the observation.

According to our linear theory, these uneven structures take the form of a sinusoidal curve. Therefore the concave and the convex part have the same form. But observation shows that the forms are different between the convex and the concave part. Namely at the growing 


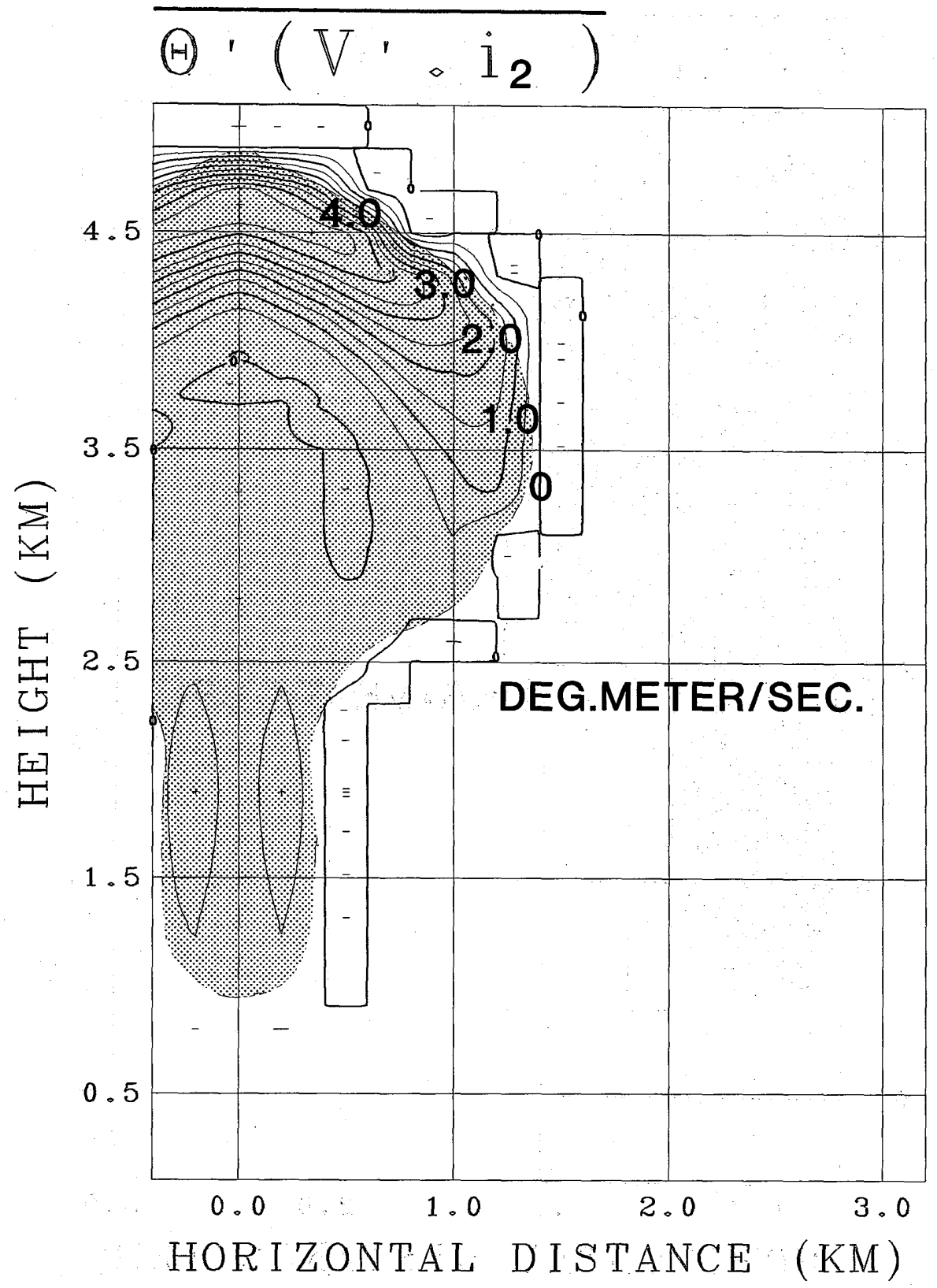

Fig. 8 The distribution of the eddy terms for the potential temperature $\overline{\left(\Theta^{\prime}\left(v^{\prime} \cdot i_{2}\right)\right)}$. The shaded region means the cloudy air $\left(Q_{c} \geqq 0.1 \mathrm{~g} / \mathrm{kg}\right)$. 


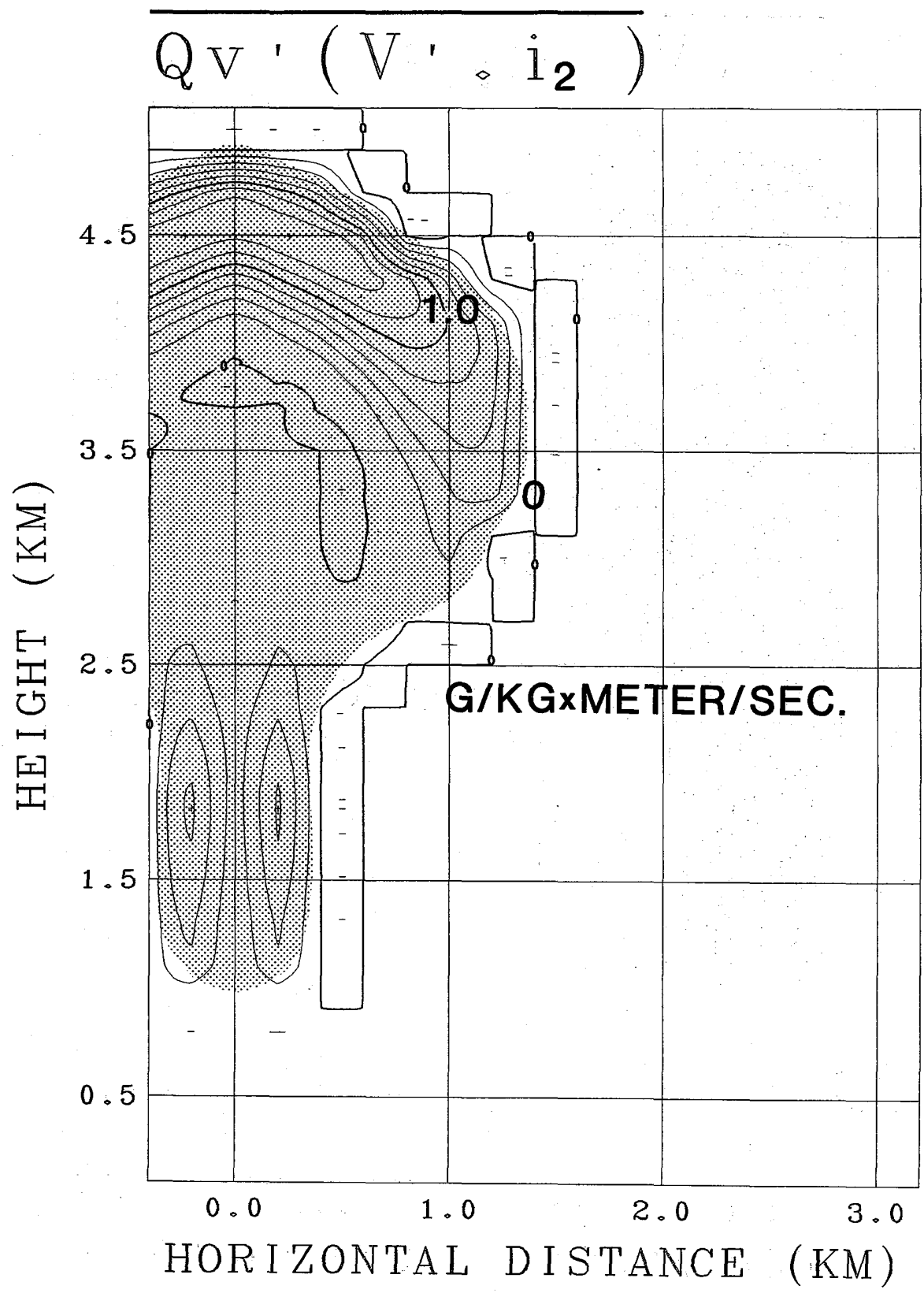

Fig. 9 The distribution of the eddy terms far the water vapor mixing ratio $\overline{\left.Q_{v}^{\prime}\left(v^{\prime} \cdot i_{2}\right)\right)}$. The shaded region means the cloudy air $\left(Q_{c} \geqq 0.1 \mathrm{~g} / \mathrm{kg}\right)$. 


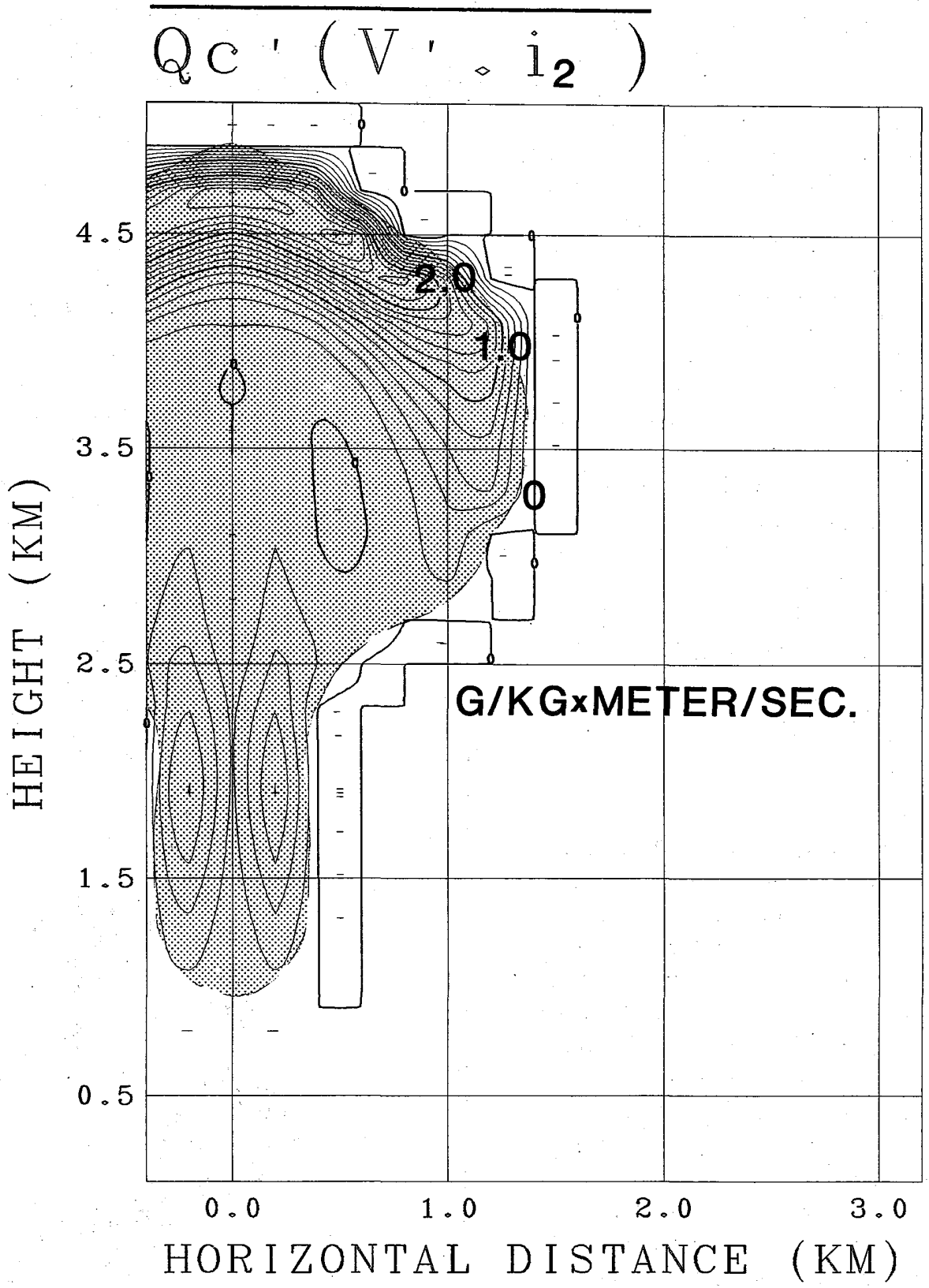

Fig. 10 The distribution of the eddy terms for the cloud water mixing ratio $\left(\overline{Q_{c}{ }^{\prime}\left(v^{\prime} \cdot i_{2}\right)}\right)$. The shaded region means the cloudy air $\left(Q_{c} \geqq 0.1 \mathrm{~g} / \mathrm{kg}\right)$. 
stage, the concave part is round and the convex part is pointed inward. At the decaying stage the situation is reversed. In order to examine this phenomenon, however, a nonlinear theory will be necessary.

We believe that our theory is no other than what Klaassen and Clark aimed at in their paper (Klaassen and Clark, 1985). They mentioned two kinds of difficulties that faced them in performing a rigorous analysis of stability. One of them is that the surface of the cloud is not stationary. We think that it can be solved by taking the spatially averaged value of the physical variables for a few hundred meters. The other one is the need to take into consideration the effect of the phase change. But our formulation has overcome this difficulty by introducing Eqs.(2.4) (2.6).

\subsection{The effect of mean wind}

In the real clouds, the value of the shear of the mean wind is of the order of $10^{-2} \mathrm{sec}^{-1}$.

In Eq.(3.3), the effect of the shear is negligible in the spatial derivative terms when

$$
t=t^{\prime} \ll 100 \mathrm{sec} \text {. }
$$

as we discussed in 3.1.

Another effect of the mean wind shear comes into the eigenvalue equation in the form of $\operatorname{div} \cdot \overrightarrow{\vec{V}}$, which is of the order of $10^{-4} \mathrm{sec}^{-1}$ and negligible compared with other terms. Therefore the prediction of this paper well expresses the behaviour of the uneven structure for about a few tens of seconds after it appeared. But as time approaches $t \simeq 100 \mathrm{sec}$., the effect of the shear ceases to be negligible. As time passes, the mode with the maximum growth-rate suffers translation and transformation. This corresponds to the observation in which the convex part appears at a certain angle to the horizontal line, but it is eventually advected and its angle to the horizontal line changes. We will discuss this in our forthcoming papers.

Further we should comment on Eq.(3.8). By making the approximation Eq.(3.7), we could obtain the plane wave solution whose wave length is spatially uniform. As time passes, the effect of the shear appears and the uniformity of the wavelength is broken. But at the place where the condition (3.8) is not available, the wavelength depends on the place from the first instant. We give a simple example of this in Appendix E. Therefore it is necessary to obtain a more exact solution without approximation (3.7), if possible.

\subsection{The fractal-like structure of convective cloud}

In our theory, only the direction of the wave number vector is fixed to determine the most unstable mode and its length are not be chosen. This suggests the fractal or self-similar structure of the surface of the convective cloud. Suppose first the boundary of the cloud with no structure, but by the instability of this stratifi-

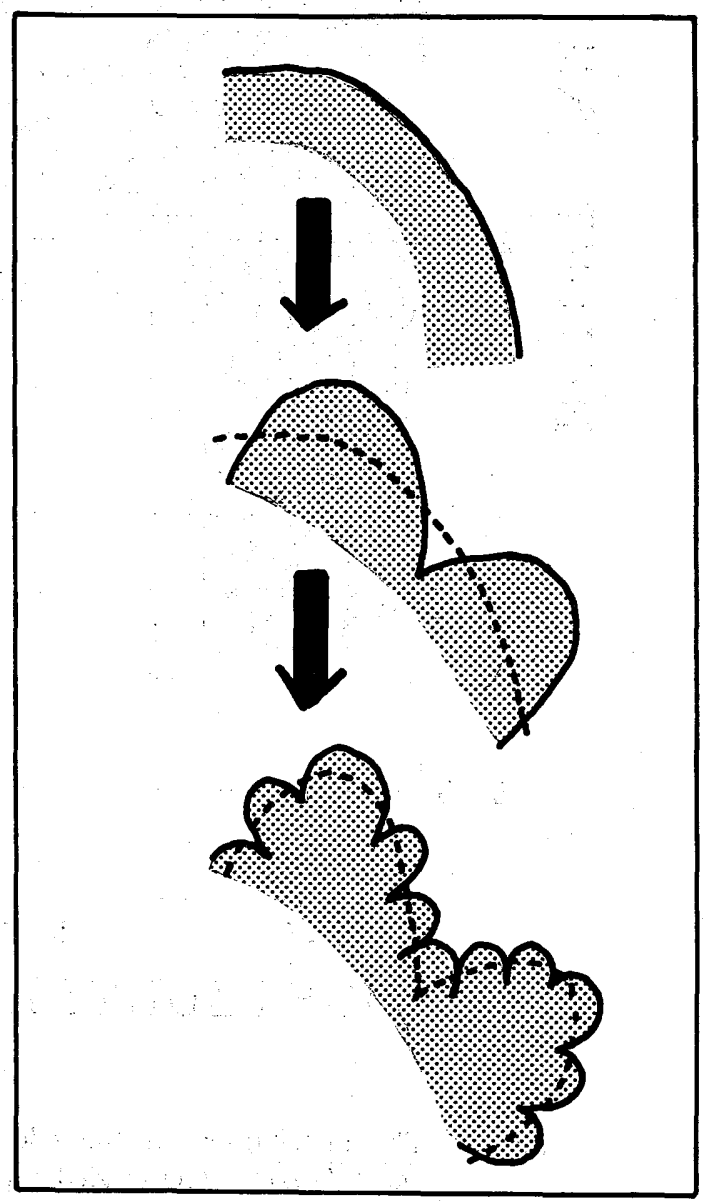

Fig. 11 Schematic explanation of how the fractal-like structure of the convective cloud is formed. 
cation, an uneven structure will appear. But this stratification is also unstable and a smaller structure will appear and so on. It is not necessary that this process should occur successively. It is possible that the phenomena of the several scales appear simultaneously.

\subsection{The reduction of our solution to the case of dry air}

Our solution in this paper reduces to the well-known dry air stability problem when we delete water substance and $L_{v}=0$ in (3.22), (3.23), (3.24) and (3.28) and the stratification of air is not tilted. Therefore the usual internal gravity wave appears when the stratification is stable. (In that case, the $\sigma$ becomes purely imaginary).

We wish to remind that our solution describes the growing stage (See 3.1). In the case of dry air, our formulation reduces to the study by Brown and Stewartson (1980) in which the plane wave solution is advected by the mean wind shear and accepts transformation. We will deal with this situation in a forthcoming paper.

Our formulation does not reduce to the solution by Kuo (1963) in which the the Richardson number appears explicitly and the pattern of the solution does not change in time. Therefore we should consider possibility of another kind of unstable solution whose pattern does not change in time under the existence of mean shear flow and water substance. But our concern is confined to the usually observed uneven structure of clouds which gets transformation under shear flow.

\section{Summary and conclusions}

We have offered a theory which can describe consistently both the mechanism which produces the uneven structure of the surface of the convective cloud and the dynamical picture of the entrainment or mixing of the cloudy air with the surrounding clear air. This theory is a generalization of conditional instability in two ways: the stratification of the humid air is tilted against the horizon and the effect of the wind shear is taken into account.
In this paper, we only used the data of a convective cloud at its growing stage. But our formulation is very general. Therefore it is possible to analyze the stability problem of clouds of other kinds and at other stages of its life if suitable data are available.

In the forthcoming paper, we will describe the tendency of the motion of the uneven structure of the surface of the convective cloud and compare the results with observation.

Acknowledgment:- The author wishes to sincerely thank Dr. J. Shiino of the Meteorological Research Institute (MRI) for giving him the opportunity of carrying out this study, together with every form of support. $\mathrm{He}$ would like to thank Dr. M. Yamasaki for his deep understanding and giving him important advice and encouragement to go ahead with this work. He also wishes to thank Dr. T. Matsuo for teaching him the existence of Einstein's formula about the molecular diffusion. Dr. T. Tanaka of MRI offered him every facility for the time lapse video recording and its analysis. Dr. J. Yano of Kyoto University gave him valuable imformation on literature concerning the fractal structure of the cloud. Mr. H. Ohnishi kindly taught him how to obtain the pictures by using the electric computer. Mr. M. Hara also kindly taught him how to use the personal computer as the word processor. He also wishes to thank Mr. Tagawa, the system engineer of HITACHI super electric computing system (S 810-10) for much kind advice about the programming. Thanks are extended to his colleagues in the Typhoon Research Division of the MRI for their kind support of this study. Finally the author wishes to express his deep appreciation to Prof. Dr. P. V. Hobbs and the members of the International Program Committee of the 10th International Cloud Physics Conference at Bad Homburg (F.R.G) for giving him an opportunity to make a presentation about this work. 
Appendix A. List of symbols

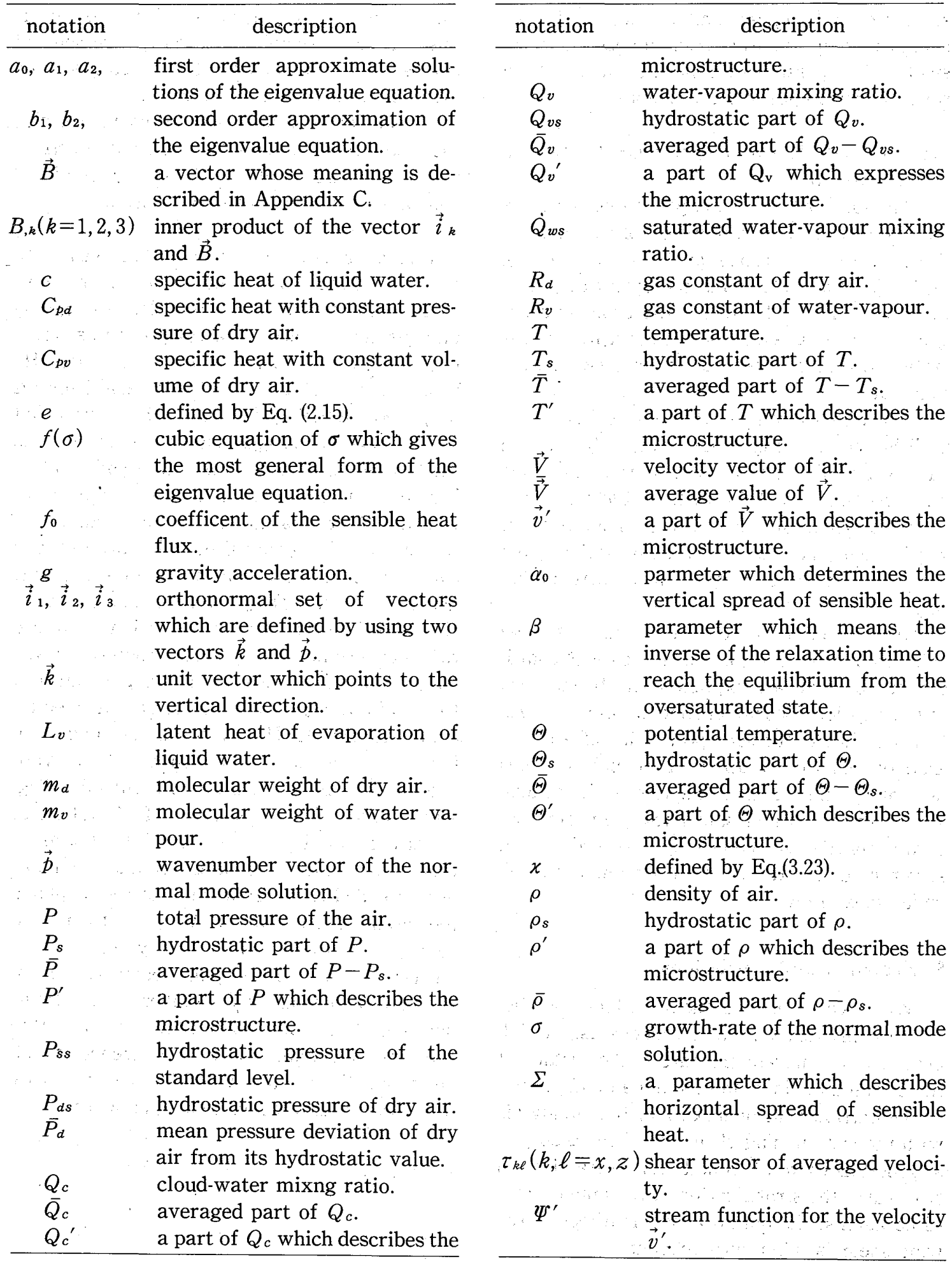


Appendix B. The physical meaning of the parameter $\beta$

In this appendix, we will clarify the physical meaning of the parameter $\beta$ which appeared in section 2.

Let us neglect the advective terms and the dependence of $\Theta$ on the pressure in Eqs.(2.4) and (2.5); then we obtain

$$
\begin{gathered}
\frac{\partial T}{\partial t}=\frac{L_{v}}{C_{p d}} \beta \frac{Q_{v}-Q_{w s}(T)}{1+\frac{L_{v}}{C_{p d}} \frac{d Q_{w s}(T)}{d T}}, \\
\frac{\partial Q_{v}}{\partial t}=-\beta \frac{Q_{v}-Q_{w s}(T)}{1+\frac{L_{v}}{C_{p d}} \frac{d Q_{w s}(T)}{d T}} .
\end{gathered}
$$

Further we neglect the dependence of $L_{v}$ on $T$. Then it is easy to see from Eqs.(B.1) and (B.2) that $C_{p d} T+L_{v} Q_{v}$ is a constant of time, which we name $C$ :

$$
C=C_{p d} T+L_{v} Q_{v} .
$$

If we substitute Eq.(B.3) into (B.1), we get

$$
\frac{\partial T}{\partial t}=\beta \frac{C-C_{p d} T-L_{v} Q_{w s}(T)}{\frac{d\left(C_{p d} T+L_{v} Q_{w s}(T)-C\right)}{d T}} .
$$

We define a new variable $K(T)$ as

$$
\begin{aligned}
K(T) & =C_{p d} T+L_{v} Q_{w s}(T)-C \\
& =L_{v}\left(Q_{w s}(T)-Q_{v}\right) .
\end{aligned}
$$

Then it satisfies

$$
\frac{\partial T}{\partial t} \frac{d K(T)}{d T}=-\beta K(T)
$$

by using Eqs.(B.4) and (B.5). This equation is no other than

$$
\frac{\partial K}{\partial t}=-\beta K\left(T_{t}\right) .
$$

This can easily be solved to give

$$
K\left(T_{t}\right)=K\left(T_{0}\right) \exp (-\beta t) .
$$

If we write the initial value of $T$ and $Q_{v}$ to be $T_{0}$ and $Q_{v_{0}}$ respectively, the solution (B.7) is rewritten as

$$
Q_{w s}(T)-Q_{v}=\exp (-\beta t)\left(Q_{w s}\left(T_{0}\right)-Q_{v 0}\right) .
$$

The physical meaning of $\beta$ at this gtage is clear. Initial oversaturation $\left(Q_{w s}\left(T_{0}\right)<Q_{v 0}\right)$, in a time of $1 / \beta$, almost goes to the equilibrium state. Therefore the $1 / \beta$ can be interpreted to be a relaxation time to settle the oversaturation. A similar interpretation can be made in the case of the undersaturation if there is plenty of cloud water.

Next we estimate the value of $\beta$. We imagine a situation in which $n_{0}$ cloud water droplets are floating in a volume of $1 \mathrm{~cm}^{3}$. Then mean distance $d$ between the droplets in $\mathrm{cm}$ unit is given by

$$
d=\left(\frac{1}{n_{0}}\right)^{1 / 3}
$$

Further we assume the existence of oversaturated water-vapour in this atmosphere. Every water-vapour molecule is supposed to travel by the diffusive process, and after it has traveled the distance $d$, the oversaturation is considered to settle into an equilibrium. Let us suppose that during the time interval $\Delta t$, a watervapour molecule travels the distance $\dot{\Delta} x$ by diffusion. Then these two quantities are known to be connected by Einstein's relation

$$
\overline{(\Delta x)^{2}}=D_{v} \Delta t,
$$

where $D_{v}$ is the diffusion coefficient for water vapour in the air and the bar in the left hand side means the mean value over many molecules. If we substitute $d$ instead of $\Delta x$ in Eq.(B.10), we obtain the relaxation time $\tau$ by

$$
\tau=\frac{1}{D_{v}} d^{2} .
$$

By the interpretation we made earlier in this appendix, we have

$$
\tau=\frac{1}{\beta} .
$$

From Eqs.(B.9), (B.11) and (B.12), we obtain the following formula for $\beta$ :

$$
\beta=\frac{D_{v}}{d^{2}}=D_{v}\left(n_{0}\right)^{2 / 3}
$$


About the value of $D_{v}$, we refer to the result of Hall and Pruppacher (1976). They obtained

$$
D_{v}\left[\mathrm{~cm}^{2} \mathrm{sec}^{-1}\right]=0.211\left(T / T_{0}\right)^{1.94}\left(P_{0} / P\right) \text {, }
$$

where $T_{0}=273.15 \mathrm{~K}$ and $P_{0}=1013.25 \mathrm{mb}$.

If we employ $n_{0}=200, P=500 \mathrm{mb}$ and $T=$ $258 \mathrm{~K}$, then we get

$$
\beta=13.1 \mathrm{sec}^{-1}
$$

In this way, we can assume that the value of $\beta$ in the typical convective cloud to be of the order of $10 \mathrm{sec}^{-1}$.

\section{Appendix C. Acquisition of buoyancy by a traveling parcel in a cloud.}

In this appendix, we study the process of the acquisition of buoyancy by a parcel travelling in a cloud in which the stratification of the physical variables are tilting nearly along the surface of the cloud.

Let us suppose that a parcel in a cloud

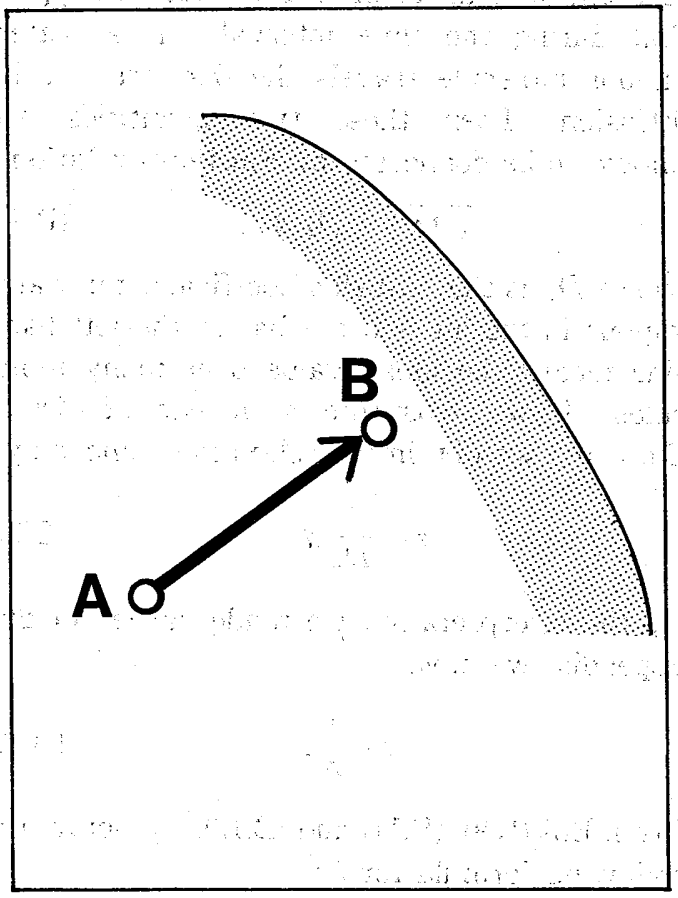

Fig. 12 Displacement of a wet air parcel in a cloud from point $\mathrm{A}$ to point $\mathrm{B}$. moves from the point $A$ to the point $B$.

After the end of this movement, the parcel had an experience of change of density $\Delta_{m} \rho$. Because of the existence of the stratification, there is a difference of density between these two points, which we write $\Delta_{s} \rho$. Then the parcel which came from the point $A$ and arrived at the point $B$ acquires buoyancy:

$$
\vec{F}_{B}=g \vec{k}\left(\Delta_{s} \rho-\Delta_{m} \rho\right) / \rho \text {. }
$$

A's the first step, we calculate $\Delta_{s} \rho$. In the process of calculation, the water-vapour pressure is assumed to be just saturated. Henceforth we use symbol $\Delta_{m}$ to show the difference of values which is caused by the travel from the point $\mathrm{A}$ to $\mathrm{B}$, and $\Delta_{s}$ to show the difference by the stratification.

The first law of thermodynamics in our problem is written as

$$
\begin{gathered}
{\left[C_{p d}+\left(Q_{w s}+Q_{c}\right) c\right] \frac{\Delta_{m} T}{T}-R_{d} \frac{\Delta_{m} P_{d}}{P_{d}}} \\
\quad+\Delta_{m}\left(\frac{L_{v} Q_{w s}}{T}\right)=0
\end{gathered}
$$

where $P_{d}$ is the pressure of the dry air. The meaning of the other symbols are shown in Appendix A. From the equation of the state of dry air and the water-vapour, we have:

$$
\begin{aligned}
& \frac{\Delta_{m} P_{d}}{P_{d}}-\frac{\Delta_{m} T}{T}-\frac{\Delta_{m} \rho_{d}}{\rho_{d}}=0, \\
& \frac{\Delta_{m} P_{v}}{P_{v}}-\frac{\Delta_{m} T}{T}-\frac{\Delta_{m} \rho_{v}}{\rho_{v}}=0
\end{aligned}
$$

where $\rho_{v}$ and $P_{v}$ are the density and the pressure of the water-vapour respectively. From the definition of $Q$ ws, we obtain

$$
\frac{\Delta_{m} Q_{w s}}{Q_{w s}}+\frac{\Delta_{m} \rho_{d}}{\rho_{d}}-\frac{\Delta_{m} \rho_{v}}{\rho_{v}}=0 .
$$

From the Clausius-Clapeyron equation, we get

$$
\frac{\Delta_{m} P_{v}}{P_{v}}-\frac{L_{v}}{R_{v} T^{2}} \Delta_{m} T=0 .
$$

From the balance of pressure between the parcel and the surrounding atmosphere we obtain 


$$
\Delta_{m} P_{d}+\Delta_{m} P_{v}=\Delta_{s} P .
$$

From (C.2) (C.7), we obtain $\Delta_{m} T, \Delta_{m} Q_{w s}$, $\Delta_{m} P_{v}, \Delta_{m} P_{d}, \Delta_{m} \rho_{v}$ and $\Delta_{m} \rho_{d}$. Here we only give $\Delta_{m} T$.

$$
\begin{aligned}
& \frac{\Delta_{m} T}{T} \\
& R_{d}\left(1+\frac{L_{v} Q_{w s}}{R_{d} T}\right) \\
& C_{p d}+\frac{L_{v}^{2} Q_{w s}}{R_{v} T^{2}}+Q_{c} c+Q_{w s} C_{p v}+\frac{L_{v}^{2} Q_{w s}^{2}}{R_{d} T^{2}} \\
& \times \frac{\Delta_{s} P}{P_{d}} .
\end{aligned}
$$

Now from the definition of $Q_{w s}$ and $Q_{c}$,

$$
\rho=\rho_{d}+\rho_{v}+\rho_{c}=\rho_{d}\left(1+Q_{w s}+Q_{c}\right),
$$

where $\rho_{c}$ is the density of the cloud water. Then from Eq.(C.9), we obtain

$$
\begin{aligned}
\frac{\Delta_{m} \rho}{\rho} & =\frac{\Delta_{m} \rho_{d}}{\rho_{d}}+\frac{\Delta_{m} Q_{w s}+\Delta_{m} Q_{c}}{1+Q_{w s}+Q_{c}} \\
& =\frac{\Delta_{m} \rho_{d}}{\rho_{d}},
\end{aligned}
$$

because in the parcel method, the total water content $Q_{w s}+Q_{c}$ must be conserved.

From the equation of the state for the moist air, we get

$$
\begin{aligned}
\frac{\Delta_{m} P}{P}= & \frac{\Delta_{m} \rho_{d}}{\rho_{d}}+\frac{\Delta_{m} T}{T}+\frac{m_{d} \Delta_{m} Q_{w s}}{m_{v}\left(1+\frac{m_{d}}{m_{v}} Q_{w s}\right)} \\
\simeq & \frac{\Delta_{m} \rho_{d}}{\rho_{d}}+\frac{\Delta_{m} T}{T}+\frac{m_{d}}{m_{v}} \Delta_{m} Q_{w s} \\
= & \frac{\Delta_{m} \rho_{d}}{\rho_{d}}+\frac{\Delta_{m} T}{T} \\
& \quad+\frac{m_{d}}{m_{v}} Q_{w s}\left(\frac{L_{v}}{R_{v} T^{2}} \Delta_{m} T-\frac{\Delta_{m} P_{d}}{P_{d}}\right) \\
= & \frac{\Delta_{m} \rho_{d}}{\rho_{d}}+\frac{\Delta_{m} T}{T}\left(1+\frac{L_{v} Q_{w s}}{R_{d} T}\right)
\end{aligned}
$$

$$
\begin{gathered}
-\frac{m_{d}}{m_{v}} Q_{w s} \frac{\Delta_{m} P_{d}}{P_{d}} \\
\simeq \frac{\Delta_{m} \rho_{d}}{\rho_{d}}+\frac{\Delta_{m} T}{T}\left(1+\frac{L_{v} Q_{w s}}{R_{d} T}\right) .
\end{gathered}
$$

Then we have

$$
\frac{\Delta_{m} \rho_{d}}{\rho_{d}} \simeq \frac{\Delta_{m} P}{P}-\frac{\Delta_{m} T}{T}\left(1+\frac{L_{v} Q_{w s}}{R_{d} T}\right) .
$$

Substituting Eq.(C.8) into (C.11) and using Eq.(C.10), we get

$$
\begin{array}{r}
\frac{\Delta_{m} \rho}{\rho}=\frac{\Delta_{m} P}{P} \\
\frac{R_{d}\left(1+\frac{L_{v} Q_{w s}}{R_{d} T}\right)^{2}}{C_{p d}+\frac{L_{v}^{2} Q_{w s}}{R_{v} T^{2}}+Q_{c} C+Q_{w s} C_{p v}+\frac{L_{v}^{2} Q_{w s}^{2}}{R_{d} T^{2}}} \\
\times \frac{\Delta_{s} P}{P_{d}} .
\end{array}
$$

As a next step, we must obtain $\Delta_{s} \rho$. From eq.(C.9), we have

$$
\begin{aligned}
\frac{\Delta_{s} \rho}{\rho} & =\frac{\Delta_{s} \rho_{d}}{\rho_{d}}+\frac{\Delta_{s} Q_{w s}+\Delta_{s} Q_{c}}{1+Q_{w s}+Q_{c}} \\
& \simeq \frac{\Delta_{s} \rho_{d}}{\rho_{d}}+\Delta_{s} Q_{w s}+\Delta_{s} Q_{c} .
\end{aligned}
$$

Again from the equation of moist air, we get

$$
\begin{aligned}
\frac{\Delta_{s} P}{P} & =\frac{\Delta_{s} \rho_{d}}{\rho_{d}}+\frac{\Delta_{s} T}{T}+\frac{m_{d} \Delta_{s} Q_{w s}}{m_{v}\left(1+\frac{m_{d}}{m_{v}} Q_{w s}\right)} \\
& \simeq \frac{\Delta_{s} \rho_{d}}{\rho_{d}}+\frac{\Delta_{s} T}{T}+\frac{m_{d}}{m_{v}} \Delta_{s} Q_{w s} .
\end{aligned}
$$

Then from Eqs.(C.13) and (C.14) we obtain

$$
\begin{aligned}
\frac{\Delta_{s} \rho}{\rho}= & \frac{\Delta_{s} P}{P}-\frac{\Delta_{s} T}{T} \\
& -\left(\frac{m_{d}}{\dot{m}_{v}}-1\right) \Delta_{s} Q_{w s}+\Delta_{s} Q_{c}
\end{aligned}
$$

By substituting Eqs.(C.12) and (C.15) into (C.1) and knowing that $\Delta_{m} P=\Delta_{s} P$, we have

$$
\vec{F}_{B}=g \vec{k}\left[-\frac{\Delta_{s} T}{T}-\left(\frac{m_{d}}{m_{v}}-1\right) \Delta_{s} Q_{w s}+\Delta_{s} Q_{c}\right.
$$




$$
\begin{gathered}
+\frac{R_{d}\left(1+\frac{L_{v} Q_{w s}}{R_{d} T}\right)^{2}}{C_{p d}+\frac{L_{v}^{2} Q_{w s}}{R_{v} T^{2}}+Q_{c} c+Q_{w s} C_{p v}+\frac{L_{v}^{2} Q_{w s}^{2}}{R_{d} T^{2}}} \\
\left.\times \frac{\Delta_{s} P}{P_{d}}\right] .
\end{gathered}
$$

Now by making the approximation

$$
\begin{gathered}
\Delta_{s} P \simeq \Delta_{s} \rho_{d}, \\
P \simeq P_{d}, \\
C_{p d}+\frac{L_{v}^{2} Q_{w s}}{R_{v} T^{2}}+Q_{c} c+Q_{w s} C_{p v}+\frac{L_{v}{ }^{2} Q_{w s}{ }^{2}}{R_{d} T^{2}} \\
\simeq C_{p d}+\frac{L_{v}{ }^{2} Q_{w s}}{R_{v} T^{2}}
\end{gathered}
$$

we obtain the final form

$$
\begin{array}{r}
\vec{F}_{B}=g \vec{k}\left[-\frac{\Delta_{s} \Theta}{\Theta}-\left(\frac{m_{d}}{m_{v}}-1\right) \Delta_{s} Q_{w s}+\Delta_{s} Q_{c}\right. \\
\left.-\frac{R_{d}}{C_{p d}} \frac{\left(\frac{L_{v}^{2}}{C_{p d} R_{v} T^{2}}-\frac{2 L_{v}}{R_{d} T}\right) Q_{w s}}{1+\frac{L_{v}^{2} Q_{w s}}{R_{v} T^{2}}} \frac{\Delta_{s} P}{P}\right] \\
\text { (C.16) }
\end{array}
$$

from $A$ to $B$, we write

$$
\Delta_{s}=\Delta \vec{r} \cdot \operatorname{grad}
$$

Then Eq.(C.16) becomes

$$
\vec{F}_{B}=g \vec{k}(\Delta \vec{r} \cdot \vec{J}),
$$

where the vector $\vec{J}$ is defined by

$$
\begin{aligned}
\vec{J}= & -\frac{\operatorname{grad} \Theta}{\Theta} \\
& -\left(\frac{m_{d}}{m_{v}}-1\right) \operatorname{grad} Q_{w s}+\operatorname{grad} Q_{c} \\
& -\frac{R_{d}}{C_{p d}} \frac{\left(\frac{L_{v}{ }^{2}}{C_{p d} R_{v} T^{2}}-\frac{2 L_{v}}{R_{d} T}\right) Q_{w s}}{1+\frac{L_{v}{ }^{2} Q_{w s}}{R_{v} T^{2}}} \frac{\operatorname{grad} P}{P} .
\end{aligned}
$$

Therefore it is clear that if the parcel travels parallel to the vector $\vec{J}$, it acquires the maximum increase of buoyancy. If it moves inversely $\vec{J}$, it experiences the maximum decrease of buoyancy.

Meanwhile, from Eqs.(3.22) (3.24) and remembering the fact that

$$
Q_{v s}+\bar{Q}_{v} \simeq Q_{w s}\left(T_{s}+\bar{T}\right),
$$

we obtain

If we denote by $\Delta \vec{r}$ the displacement vector

$$
\begin{aligned}
\vec{B}=\frac{1}{\Theta_{s}+\bar{\Theta}} \vec{\nabla}\left(\Theta_{s}+\bar{\Theta}\right)+\left(\frac{m_{d}}{m_{v}}-1\right) \vec{\nabla} Q_{w s}\left(T_{s}+\bar{T}\right)-\vec{\nabla} \bar{Q}_{c} \\
+\frac{\left(\frac{L_{v}}{C_{p d}\left(T_{s}+\bar{T}\right)}-\frac{m_{d}}{m_{v}}\right)}{\left(1+\frac{L_{v}{ }^{2} Q_{w s}}{C_{p d} R_{v}\left(T_{s}+\bar{T}\right)^{2}}\right)}\left[\vec{\nabla} Q_{w s}-\left(\frac{d Q_{w s}}{d T}\right)_{T=T_{s}+\bar{T}} \vec{\nabla}\left(\Theta_{s}+\bar{\Theta}\right)\left(\frac{P_{d s}+P_{d}}{P_{s s}}\right)^{e}\right]
\end{aligned}
$$

Here we define explicitly the meaning of $\Theta_{s}$ $+\bar{\Theta}$ by the following equation :

$$
\Theta_{s}+\bar{\Theta}=\left(T_{s}+\bar{T}\right)\left(\frac{P_{d s}+P_{d}}{P_{s s}}\right)^{e} .
$$

The equation in the bracket of the fourth term of the righthand side of (C.20) is rewritten as

$$
\vec{\nabla} Q_{w s}-\left(\frac{d Q_{w s}}{d T}\right)_{T=T_{s}+\bar{T}} \vec{\nabla}\left(\Theta_{s}+\bar{\Theta}\right)\left(\frac{P_{d s}+P_{d}}{P_{s s}}\right)^{e}
$$




$$
\begin{aligned}
& =\vec{\nabla}\left(\frac{P_{v s}+\bar{P}_{v}}{P_{d s}+\bar{P}_{d}} \frac{R_{d}}{R_{v}}\right)-\left(\frac{d Q_{w s}}{d T}\right)_{T=T_{s}+\bar{T}} \vec{\nabla}\left(\Theta_{s}+\bar{\Theta}\right)\left(\frac{P_{d s}+P_{d}}{P_{s s}}\right)^{e} \\
& =-\frac{1}{P_{d s}+\bar{P}_{d}} \vec{\nabla}\left(P_{d s}+\bar{P}_{d}\right) Q_{w s}+\left[\vec{\nabla}\left(T_{s}+\bar{T}\right)-\vec{\nabla}\left(\Theta_{s}+\bar{\Theta}\right)\left(\frac{P_{d s}+P_{d}}{P_{s s}}\right)^{e}\right] \frac{d Q_{w s}}{d T} \\
& =\frac{1}{P_{d s}+\bar{P}_{d}} \vec{\nabla}\left(P_{d s}+\bar{P}_{d}\right)\left[\frac{R_{d} L_{v}}{C_{p d} R_{v}\left(T_{s}+\bar{T}\right)}-1\right] Q_{w s} .
\end{aligned}
$$

Then this is substituted into (C.20) giving

$$
\begin{aligned}
\vec{B}=\frac{1}{\Theta_{s}+\bar{\Theta}} \vec{\nabla}\left(\Theta_{s}+\bar{\Theta}\right)+\left(\frac{m_{d}}{m_{v}}-1\right) \vec{\nabla} Q_{w s}\left(T_{s}+\bar{T}\right)-\vec{\nabla} \bar{Q}_{c} \\
+\frac{Q_{w s}}{P_{d s}+\bar{P}_{d}} \vec{\nabla}\left(P_{d s}+\bar{P}_{d}\right) \frac{\left(\frac{L_{v}}{C_{p d}\left(T_{s}+\bar{T}\right)}-\frac{m_{d}}{m_{v}}\right)\left(\frac{R_{d} L_{v}}{C_{p d} R_{v}\left(T_{s}+\bar{T}\right)}-1\right)}{\left(1+\frac{L_{v}{ }^{2} Q_{w s}}{C_{p d} R_{v}\left(T_{s}+\bar{T}\right)^{2}}\right)}
\end{aligned}
$$

The numerator of the last term in Eq.(C.22) is approximated to be

$$
\left(\frac{L_{v}}{C_{p d}\left(T_{s}+\bar{T}\right)}-\frac{m_{d}}{m_{v}}\right)\left(\frac{R_{d} L_{v}}{C_{p d} R_{v}\left(T_{s}+\bar{T}\right)}-1\right) \simeq \frac{R_{d}}{C_{p d}}\left(\frac{L_{v}{ }^{2}}{C_{p d} R_{v}\left(T_{s}+\bar{T}\right)^{2}}-\frac{2 L_{v}}{R_{d}\left(T_{s}+\bar{T}\right)}\right)
$$

Then we obtain the final result

$$
\begin{aligned}
\vec{B}=\frac{1}{\Theta_{s}+\bar{\Theta}} \vec{\nabla}\left(\Theta_{s}+\bar{\Theta}\right)+\left(\frac{m_{d}}{m_{v}}-1\right) \vec{\nabla} Q_{w s}\left(T_{s}+\bar{T}\right)-\vec{\nabla} \bar{Q}_{c} \\
+\frac{Q_{w s}}{P_{d s}+\bar{P}_{d}} \vec{\nabla}\left(P_{d s}+\bar{P}_{d}\right) \frac{\left(\frac{L_{v}{ }^{2}}{C_{p d} R_{v}\left(T_{s}+\bar{T}\right)^{2}}-\frac{2 L_{v}}{R_{d}\left(T_{s}+\bar{T}\right)}\right)}{\left(1+\frac{L_{v}{ }^{2} Q_{w s}}{C_{p d} R_{v}\left(T_{s}+\bar{T}\right)^{2}}\right)} \frac{R_{d}}{C_{p d}}
\end{aligned}
$$

where the following approximations are used:

$$
\begin{aligned}
P_{d s} \simeq P_{s}, \quad P_{d} \simeq P, \quad \vec{\nabla} P_{d s} \simeq \vec{\nabla} P_{s} \\
\text { and } \vec{\nabla} P_{d} \simeq \vec{\nabla} P .
\end{aligned}
$$

Now comparing Eq.(C.19) and (C.23) and putting $\Theta_{s}+\bar{\Theta}$ by $\Theta, \bar{Q}_{c}$ by $Q_{c}, T_{s}+\bar{T}$ by $T$ and $P_{s}$ $+\bar{P}$ by $P$, we can easily see

$$
\vec{J}=-\vec{B} \text {. }
$$

This is exactly the result we needed.

\section{Appendix D. Fstimation of the criterion (3.8)}

In this appendix we estimate the criterion (3.8) by using the same data as we used in obtaining Fig. 6.

We define the following four parameters:

$$
\begin{aligned}
& R_{x x}=\left(\tau_{x x} \tau_{x x}+\tau_{x z} \tau_{z x}\right) /\left(\tau_{x x} \bar{V}_{x 0}+\tau_{x z} \bar{V}_{z 0}\right), \\
& R_{x z}=\left(\tau_{x x} \tau_{x z}+\tau_{x z} \tau_{z z}\right) /\left(\tau_{x x} \bar{V}_{x 0}+\tau_{x z} \bar{V}_{z 0}\right),
\end{aligned}
$$
and

$$
\begin{aligned}
& R_{z x}=\left(\tau_{z x} \tau_{x x}+\tau_{z z} \tau_{z x}\right) /\left(\tau_{z x} \bar{V}_{x 0}+\tau_{z z} \bar{V}_{z 0}\right), \\
& R_{z z}=\left(\tau_{z x} \tau_{x z}+\tau_{z z} \tau_{z z}\right) /\left(\tau_{z x} \bar{V}_{x 0}+\tau_{z z} \bar{V}_{z 0}\right) .
\end{aligned}
$$

In Eq.(3.8), one of the component of the term $\bar{V}_{i} \tau_{j i}$ is

$$
\begin{aligned}
\tau_{x x} \bar{V}_{x 0}+\tau_{x z} \bar{V}_{z 0}+\left(\tau_{x x} \tau_{x x}+\tau_{x z} \tau_{z x}\right) x \\
+\left(\tau_{x x} \tau_{x z}+\tau_{x z} \tau_{z z}\right) z
\end{aligned}
$$

If approximation (3.8) is available, it should be nearly equal to 


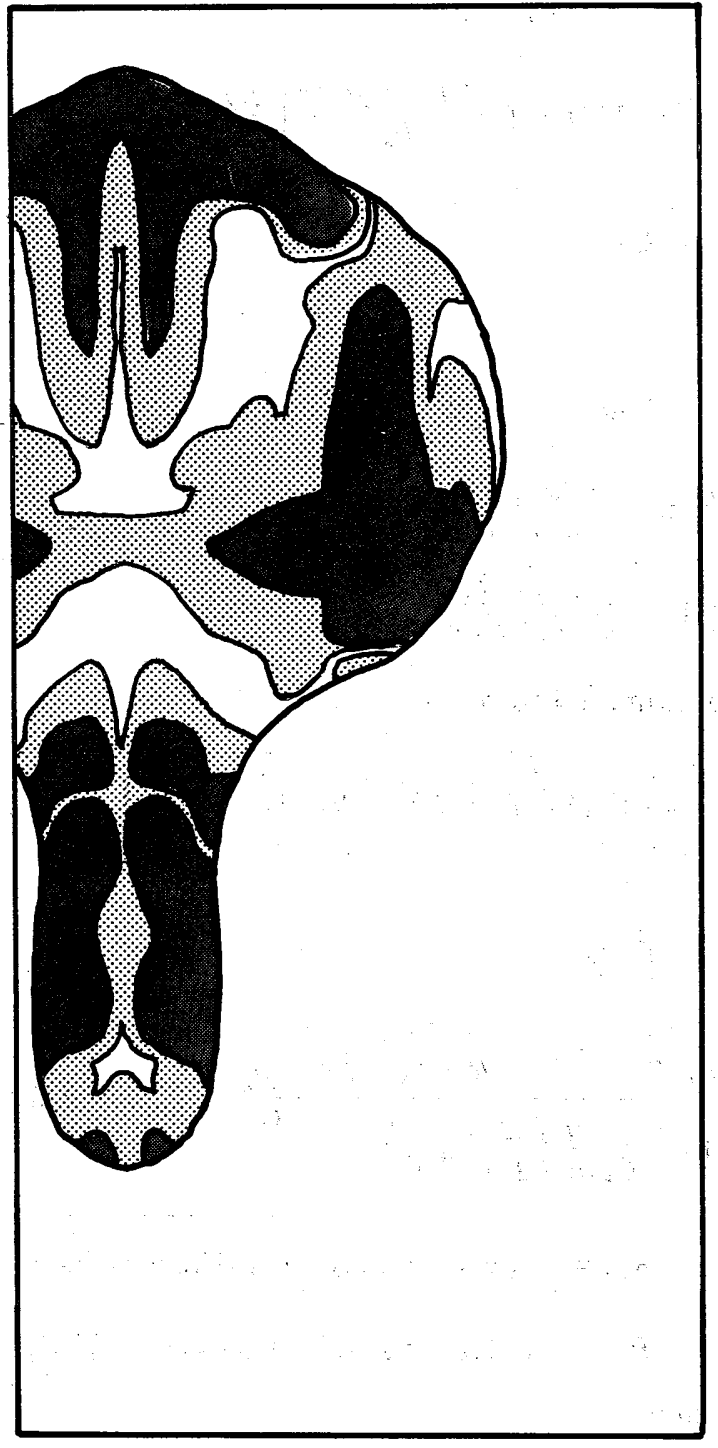

Fig. 13 Estimation of the magnitude of the four parameters defined in Appendix D for the numerically simulated cloud which is the same cloud as that in Fig. 5 . In the darkly shaded region, at least one of the four parameters has values which are more than $0.005 \mathrm{~m}^{-1}$. In the lightly shaded region, the corresponding value is $0.001 \mathrm{~m}^{-1}$.

$$
\tau_{x x} \bar{V}_{x 0}+\tau_{x z} \bar{V}_{z 0}
$$

In a subregion, the absolute value of $x$ or $z$ changes by $100 \sim 200$ meters. Therefore for this component, the following conditions are necessary:

$$
\left|R_{x x}\right| \ll 0.01 \mathrm{~m}^{-1}
$$

and

$$
\left|R_{x z}\right| \ll 0.01 \mathrm{~m}^{-1} \text {. }
$$

In Fig. 13, we show the region where one of the four parameters has absolute values larger than $0.001 \mathrm{~m}^{-1}$ (lightly shaded region) and $0.005 \mathrm{~m}^{-1}$ (darkly shaded region). This result shows that our approxion is not good. We think that the effect of the negleced term contributes to changing the wavelength place by place, and it is not important for the analysis of the instability. But this problem should be examined elsewhere.

\section{Appendix E. The effect of the shear in one-dimensional case.}

In this appendix we discuss the effect of the shear in the one-dimensional case.

Take the following simple equation:

$$
\frac{\partial f}{\partial t}+V \frac{\partial f}{\partial x}=\sigma f
$$

where

$$
\sigma>0
$$

and

$$
V=V_{0}+\alpha x \quad(\alpha>0,-\infty<x<\infty) .
$$

The parameter $\sigma$ is a growth-rate of the instability.

The general solution for this equation is

$$
f=\exp (\sigma t) g\left[t-\frac{1}{\alpha} \ln \left(1+\frac{\alpha x}{V_{0}}\right)\right],
$$

where $g$ is any function.

This solution expresses a propagating wave whose phase velocity changes from place to place and the amplitude grows exponentially. The phase velocity at $x_{0}$ is given by 


$$
c=V_{0}+\alpha x_{0} .
$$

Moreove if this solution has a single angular frequency $\omega$, the local wavelength becomes at $x=x_{0}$

$$
\lambda=\frac{2 \pi\left(V_{0}+\alpha x_{0}\right)}{\omega} .
$$

And if we consider a solution with a definite wavelength, the form of the solution will be for example

$$
f=\exp (\sigma t) \sin \left[k\left(x+\frac{V_{0}}{\alpha}\right) \exp (-\alpha t)\right] .
$$

In this case the wavelength is constant in space but it becomes larger as time passes.

Now we consider the next transformation for the coordinate which corresponds to Eq.(3.1):

$$
x^{\prime}=x-\left(V_{0}+\alpha x\right) t
$$

and

$$
t^{\prime}=t .
$$

The transformation for the derivative operators are given by

$$
\frac{\partial}{\partial x}=\left(1-\alpha t^{\prime}\right) \frac{\partial}{\partial x^{\prime}}
$$

and

$$
\frac{\partial}{\partial t}=\frac{\partial}{\partial t^{\prime}}-\left(V_{0}+\alpha x\right) \frac{\partial}{\partial x^{\prime}} .
$$

The inverse relations of Eq.(E.7) and (E.8) are

$$
x=\frac{x^{\prime}+V_{0} t^{\prime}}{1-\alpha t^{\prime}}
$$

and

$$
t=t^{\prime} \text {. }
$$

Then we obtain the following equation:

$$
V_{0}+\alpha x=\frac{V_{0}+\alpha x^{\prime}}{1-\alpha t^{\prime}} .
$$

By using these new variables, Eq.(E.1) is rewritten as

$$
\frac{\partial f}{\partial t^{\prime}}-\left(V_{0}+\alpha x\right) \alpha t^{\prime} \frac{\partial f}{\partial x^{\prime}}=\sigma f .
$$

Then Eq.(E.13) is rewritten by using (E.12)

$$
\frac{\partial f}{\partial t^{\prime}}-\alpha t^{\prime} \frac{V_{0}+\alpha x^{\prime}}{1-\alpha t^{\prime}} \frac{\partial f}{\partial x^{\prime}}=\sigma f .
$$

The general solution of this equation becomes

$$
\begin{array}{r}
f=\exp (\sigma t) g\left[t^{\prime}+\frac{1}{\alpha} \ln \left(1-\alpha t^{\prime}\right)\right. \\
\left.-\frac{1}{\alpha} \ln \left(1+\frac{\alpha x^{\prime}}{V_{0}}\right)\right] .
\end{array}
$$

It is easy to see that solution is equal to that in (E.3).

If we assume

$$
\alpha t^{\prime} \ll 1
$$

and

$$
\left|V_{0}\right| \ll\left|\alpha x^{\prime}\right|,
$$

then Eq.(E.15) is approximated to be

$$
f=\exp (\sigma t) g\left[-\frac{\alpha}{2} t^{\prime 2}-\frac{x^{\prime}}{V_{0}}\right] .
$$

This is a general solution of the next equation which is an approximated form of (E.14):

$$
\frac{\partial f}{\partial t^{\prime}}-\alpha t^{\prime} V_{0} \frac{\partial f}{\partial x^{\prime}}=\sigma f .
$$

This approximation corresponds to that in Eq.(3.8).

The solutions (E.16) and (E.15) both have a propagating property except the factor exp $(\sigma t)$. The difference is that the wavenumber is not constant in space and time in (E.15), while in (E.16) it is constant.

By this example we guess that the approximation (3.8) does not hide another instability from our study.

\section{References}

Brown, S. N. and K. Stewartson, 1980: On the algebraic decay of disturbances in a stratified shear flow, J. Fluid. Mech. 100, 811-816.

Hall, W. D. and H. R. Pruppacher, 1976: The sur- 
vival of ice particles falling from cirrus clouds in subsaturated air, J. Atmos. Sci, 33, 1995-2006.

Ichimura, I., 1988: private communication.

Klaassen, G. P. and T. L. Clark, 1985: Dynamics of the cloud-environmental interface and entrainment in small cumuli:- Two-dimensional simulation in the absence of ambient shear, $J$. Atmos. Sci., 42, 2621-2642.

Kuo, H. L., 1963: Perturbations of plane Couette flow in stratified fluid and origin of cloud streets, The Phys. of Fluids, 6, 195-211.

Paluch, I. R. and D. G. Baumgardner, 1989: Entrainment and fine-scale mixing in a continental convective cloud, J. Atmos. Sci., 46, 261-278.

Reuter, G. W. and M. K. Yau, 1987: Mixing mechanism in cumulus congestus clouds. Part I;
Observations, J. Atmos. Sci., 44, 781-797. ibid. Part II ; Numerical simulations, J. Atmos. Sci., 44, 7,98-827.

Soong, S. T. and Y. Ogura, 1973: A comparison between axisymmetric and slab-symmetric cumulus cloud models, J. Atmos. Sci., 30, 879-893.

Turner, J. S., 1986: Turbulent entrainment: the development of the entrainment assumption, and its application to geophysical flows, $J$. Fluid Mech., 173; 431-471.

Warner, J., 1969: The microstructure of cumulus cloud. Part I, General features of droplet spectrum, J. Atmos. Sci., 26, 1049-1059.

Wilhelmson, R. and Y. Ogura, 1972: The pressure perturbation and numerical modeling of a cloud, J. Atmos. Sci., 29, 1295-1307.

\section{対流雲における局所不安定論}

\section{高谷美正}

対流雲に於ける表面の凹凸の成因と, エントレインメントまたは混合の力学的構造を理解するために, 線形不 安定理論を提出する。

雲の内部では, 気圧, 温度, 水蒸気量, 雲水量の成層は水平から傾いている。そして静力学的に安定ではない ので風が吹いている。

このような場の中である種の力学的不安定が存在することが示される。これは，鉛直にのみ成層構造があり， 静止している湿潤大気に拈いて見いだされている条件付き不安定の一般化である。

雲の中でこの不安定波動が発生すると，雲水量の不均一が生じてそれが雲の凹凸に反映される。 この不安定波動による熱フラックス，水蒸気フラックス，雲水フラックスを計算すると雲の外側へ向かって正 であり，雲が薄められることがわかる。これをもってェントレインメントまたは混合のメカニズムと考斊

同時にこの不安定理論を用いて，雲内部の渦払散項に対するモデルが提案される。 